\title{
Refinement Checking on Parametric Modal Transition Systems
}

\author{
Nikola Beneš · Jan Křetínský • Kim \\ G. Larsen · Mikael H. Møller • Salomon \\ Sickert · Jiří Srba
}

the date of receipt and acceptance should be inserted later

\begin{abstract}
Modal transition systems (MTS) is a well-studied specification formalism of reactive systems supporting a step-wise refinement methodology. Despite its many advantages, the formalism as well as its currently known extensions are incapable of expressing some practically needed aspects in the refinement process like exclusive, conditional and persistent choices. We introduce a new model called parametric modal transition systems (PMTS) together with a general modal refinement notion that overcomes many of the limitations. We investigate the computational complexity of modal and thorough refinement checking on PMTS and its subclasses and provide a direct encoding of the modal refinement problem into quantified Boolean formulae, allowing us to employ state-of-the-art QBF solvers for modal refinement checking. The experiments we report on show that the feasibility of refinement checking is more influenced by the degree of nondeterminism rather than by the syntactic restrictions on the types of formulae allowed in the description of the PMTS.
\end{abstract}

\section{Introduction}

The specification formalism of Modal Transition Systems (MTS) 4, 42] grew out of a series of attempts to achieve a flexible and easy-to-use compositional development methodology for reactive systems. In fact, the formalism of MTS may be seen as a fragment of a temporal logic [9, 22, while having a behavioural semantics allowing for an easy composition with respect to process constructs.

Nikola Beně̌

Faculty of Informatics, Masaryk University, Brno, Czech Republic

Jan Křetínský

IST, Austria

Kim G. Larsen · Mikael H. Møller · Jiří Srba

Department of Computer Science, Aalborg University, Denmark

Salomon Sickert

Technical University Munich, Germany 
In short, MTS are labelled transition systems equipped with two types of transitions: must transitions which are mandatory for any implementation, and may transitions which are optional for an implementation. Refinement of an MTS now essentially consists of iteratively resolving the unsettled status of may transitions: either by removing them or by turning them into must transitions.

It is well admitted (see e.g. 52]) that MTS and their extensions like disjunctive MTS (DMTS) 43, 1-selecting MTS (1MTS) 29 and transition systems with obligations (OTS) 12 provide strong support for a specification formalism allowing for step-wise refinement process. Moreover, the MTS formalisms have applications in other contexts, which include verification of product lines [33, 41, interface theories [52, 54 and modal abstractions in program analysis 31, 34, 48.

Unfortunately, all of these formalisms lack the capability to express some intuitive specification requirements like exclusive choice (either this option or that option but not both at the same time), conditional choice (under certain conditions an option is mandatory) and persistent choice (once we commit to some option, the same choice must be taken also everywhere else). In this paper, we extend considerably the expressiveness of MTS and its variants so that it can model arbitrary Boolean conditions on transitions and also allows to instantiate persistent transitions. Our model, called parametric modal transition systems (PMTS), is equipped with a finite set of parameters that are fixed prior to the instantiation of the transitions in the specification. The generalised notion of modal refinement is designed to handle the parametric extension and it specialises to the well-studied modal refinements on all the subclasses of our model like MTS, disjunctive MTS and MTS with obligations.

To the best of our knowledge, this is the first sound attempt to introduce persistence into a specification formalism based on modal transition systems. A related work by Fecher and Schmidt on 1-selecting MTS [29] allows to model exclusive-or and the authors briefly mention the desire to extend the formalism with persistence. However, as in detail explained in the appendix of [10, their definition does not capture the intended notion of persistence. Our formalism is in several aspects semantically more general and handles persistence in a complete and uniform manner.

Our contribution can be summarised as follows:

- In Section 2, we introduce the formalism of PMTS.

- Section 3 discusses several possible refinement notions and their relationship.

- In Section 4 we provide a comprehensive complexity characterisation of modal refinement checking on a number of practically relevant subclasses of PMTS. We show that the complexity ranges from $\mathrm{P}$-completeness to $\Pi_{4}^{\mathrm{P}}$-completeness, depending on the requested generality of the PMTS specifications on the left-hand and right-hand sides.

- In Section 5, due to the high complexity of modal refinement in many cases, we also focus on its practical complexity. We reduce modal refinement problems to satisfiability problems solvable directly by QBF solvers and perform preliminary experiments indicating that our solution scales well in the number of parameters.

Related work Over the years, many extensions of MTS have been proposed. While MTS can only specify whether or not a particular transition is required, some 
extensions equip MTS with more general abilities to describe what combinations of transitions are possible. Disjunctive MTS (DMTS) [19, 43] can specify that at least one of a given set of transitions is present. One selecting MTS 29] allow to choose exactly one of them. Underspecified transition systems (UTS) 30, also allow disjunctions on may transitions. Transition systems with obligations (OTS) 12 can express positive Boolean combinations. The subclass of parameter-free PMTS we study in this paper covers all Boolean combination of transitions. The same holds for acceptance automata [50] and Boolean formulae with states [9], which both express the requirement by listing all possible sets instead of a Boolean formula. Parametric MTS (PMTS) introduced here add parameters on top of it, so that we can also express persistent choices of transitions and relate possible choices in different parts of a system. This way, one can model hardware dependencies of transitions and systems with prices [15].

There are various other approaches to deal with component refinements. They range from subtyping [44] over Java modelling language [35] to interface theories close to MTS such as interface automata [2, 45]. Similarly to MTS, interface automata are behavioural interfaces for components. However, their composition works very differently. Furthermore, their notion of refinement is based on alternating simulation [3], which has been proved in [40] strictly less expressive than MTS refinement. The compositionality of the combination of MTS and interface automata based on I/O automata [46] is further investigated in [51].

Further, alternatively to the design of correct software where an abstract verified MTS is transformed into a concrete implementation, one can consider checking correctness of software through abstracting a concrete implementation into a coarser system. The use of MTS as abstractions has been advocated e.g. in [31. While usually overapproximations (or underapproximations) of systems are constructed and thus only purely universal (or existential) properties can be checked, 31. shows that using MTS one can check mixed formulae (arbitrarily combining universal and existential properties) and, moreover, at the same cost as checking universal properties using traditional conservative abstractions. This advantage has been investigated also in the context of systems equivalent or closely related to MTS [24, 26, 27, 32, 34, 47] but parameters have not been used in this context.

The MTS framework has been extended not only with respect to the expressive power in the classical setting of automata, but also lifted to quantitative settings. This includes probabilistic systems 23 as well as various timed systems [7, 15. 20, 25, 28, 36] with clear applications in the embedded systems design. The MTS formalism can also be viewed as a fragment of mu-calculus that is "graphically representable" 9, 21. The graphical representability of a variant of alternating simulation called covariant-contravariant simulation has been recently studied in [1].

This article is an extended version of two conference papers [14, 38] with complete proofs and closing the open problems mentioned in [14.

\section{Parametric Modal Transition Systems}

In this section we present the formalism of parametric modal transition systems (PMTS), starting with a motivating example and continuing with the formal definitions, followed by the general notion of modal refinement. 


\subsection{Motivation}

Modal transition systems and their extensions described in the literature are lacking the capability to express several specification requirements like exclusive, conditional and persistent choices. We shall now discuss these limitations on an example as a motivation for the introduction of parametric MTS formalism with general Boolean conditions in specification requirements.

Consider a simple specification of a traffic light controller that can be at any moment in one of the four predefined states: red, green, yellow or yellowRed. The requirements of the specification are: when green is on the traffic light it may either change to red or yellow and if it turned yellow it must go to red afterwards; when red is on it may either turn to green or yellowRed, and if it turned yellowRed (as is the case in some countries) it must go to green afterwards.

Figure 2.1a shows an obvious MTS specification (defined formally later on) of the proposed specification. The transitions in the standard MTS formalism are either of type may (optional transitions depicted as dashed lines) or must (required transitions depicted as solid lines). In Figure 2.1c Figure 2.1d and Figure 2.1e we present three different implementations of the MTS specification where there are no more optional transitions. The implementation $I_{1}$ does not implement any may transition as it is a valid possibility to satisfy the specification $S_{1}$. Of course, in our concrete example, this means that the light is constantly green and it is clearly an undesirable behaviour that cannot be, however, easily avoided. The second implementation $I_{2}$ on the other hand implements all may transitions, again a legal implementation in the MTS methodology but not a desirable implementation of a traffic light as the next action is not always deterministically given. Finally, the implementation $I_{3}$ of $S_{1}$ illustrates the third problem with the MTS specifications, namely that the choices made in each turn are not persistent and the implementation alternates between entering yellow or not. None of these problems can be avoided when using the MTS formalism.

A more expressive formalism of disjunctive modal transition systems (DMTS) can overcome some of the above mentioned problems. A possible DMTS specification $S_{2}$ is depicted in Figure 2.1b. Here the ready and stop transitions, as well as ready and go ones, are disjunctive, meaning that it is still optional which one is implemented but at least one of them must be present. Now the system $I_{1}$ in Figure 2.1c is not a valid implementation of $S_{2}$ any more. Nevertheless, the undesirable implementations $I_{2}$ and $I_{3}$ are still possible and the modelling power of DMTS is insufficient to eliminate them.

Inspired by the recent notion of transition systems with obligations [12, we can model the traffic light using specification as a transition system with arbitrary ${ }^{1}$ obligation formulae. These formulae are Boolean propositions over the outgoing transitions from each state, whose satisfying assignments yield the allowed combinations of outgoing transitions. A possible specification called $S_{3}$ is given in Figure 2.1f and it uses the operation of exclusive-or (which is defined via negation). We will follow an agreement that whenever the obligation function for some node is not listed in the system description then it is implicitly understood as requiring all the available outgoing transitions to be present. Due to the use of exclusive-or in the obligation function, the transition systems $I_{1}$ and $I_{2}$ are not valid implementations

\footnotetext{
1 In the transition systems with obligations only positive Boolean formulae are allowed.
} 


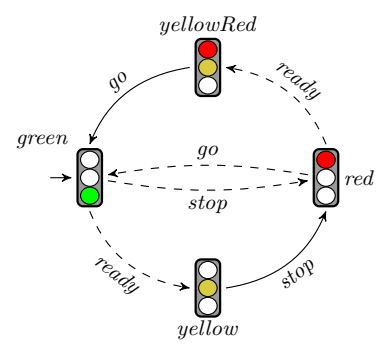

(a) MTS specification $S_{1}$

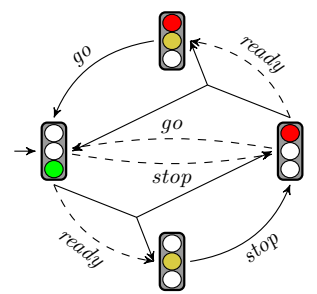

(b) DMTS specification $S_{2}$

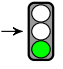

(c) Implementation $I_{1}$

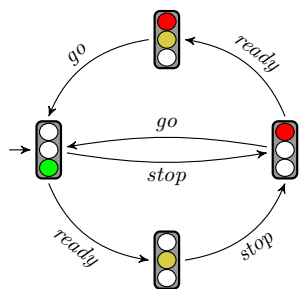

(d) Implementation $I_{2}$

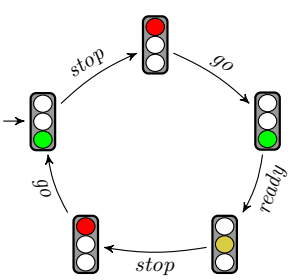

(e) Implementation $I_{3}$

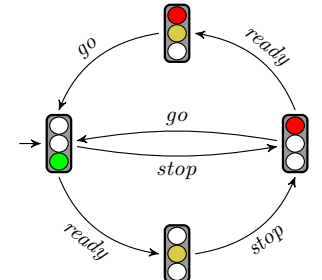

Obligation function:

$\Phi($ green $)=($ stop, red $) \oplus($ ready, yellow $)$

$\Phi($ red $)=($ go, green $) \oplus($ ready, yellowRed $)$

(f) Specification $S_{3}$

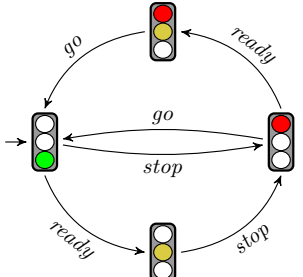

Parameters: $\{$ req YfromR, req Yfrom $G\}$

Obligation function:

$\Phi($ green $)=(($ stop, red $) \oplus($ ready, yellow $))$ $\wedge($ req $Y$ from $G \Leftrightarrow($ ready, yellow $))$

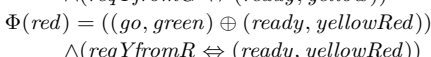

(g) PMTS specification $S_{4}$

Fig. 2.1: Specifications and implementations of a traffic light controller

any more. Nevertheless, the implementation $I_{3}$ in Figure $2.1 \mathrm{e}$ cannot be avoided in this formalism either.

Finally, the problem with the alternating implementation $I_{3}$ is that we cannot enforce in any of the above mentioned formalisms a uniform (persistent) implementation of the same transitions in all its states. In order to overcome this problem, we propose the so-called parametric MTS where we can, moreover, choose persistently whether the transition to yellow is present or not via the use of parameters. The PMTS specification with two parameters req Yfrom $R$ and req Yfrom $G$ is shown in Figure 2.1g. Fixing a priori the (Boolean) values of the parameters makes the choices permanent in the whole implementation, hence we eliminate also the last problematic implementation $I_{3}$. 
2.2 Definition of Parametric Modal Transition Systems

We shall now formally capture the intuition behind parametric MTS introduced above. First, we recall the standard propositional logic.

A Boolean formula over a set $X$ of atomic propositions is given by the following abstract syntax

$$
\varphi::=\mathbf{t t}|x| \neg \varphi|\varphi \wedge \psi| \varphi \vee \psi
$$

where $x$ ranges over $X$. The set of all Boolean formulae over the set $X$ is denoted by $\mathcal{B}(X)$. Let $\mu \subseteq X$ be a truth assignment, i.e. a set of variables with value true, then the satisfaction relation $\mu \mid=\varphi$ is given by $\mu|=\mathbf{t t}, \mu|=x$ iff $x \in \mu$, and the satisfaction of the remaining Boolean connectives is defined in the standard way. We also use the standard derived operators like exclusive-or $\varphi \oplus \psi=(\varphi \wedge \neg \psi) \vee(\neg \varphi \wedge \psi)$, implication $\varphi \Rightarrow \psi=\neg \varphi \vee \psi$ and equivalence $\varphi \Leftrightarrow \psi=(\neg \varphi \vee \psi) \wedge(\varphi \vee \neg \psi)$.

We can now proceed with the definition of parametric MTS.

Definition 2.1 A parametric MTS (PMTS) over an action alphabet $\Sigma$ is a tuple $(S, T, P, \Phi)$ where $S$ is a set of states, $T \subseteq S \times \Sigma \times S$ is a transition relation, $P$ is a finite set of parameters, and $\Phi: S \rightarrow \mathcal{B}((\Sigma \times S) \cup P)$ is an obligation function over the atomic propositions containing outgoing transitions and parameters. We implicitly assume that whenever $(a, t)$ appears in $\Phi(s)$ then $(s, a, t) \in T$. By $T(s)=$ $\{(a, t) \mid(s, a, t) \in T\}$ we denote the set of all outgoing transitions of $s$.

Note that we are not explicitly pointing to any initial state in a given PMTS. In what follows, the initial states will be clear from the context. We also recall the agreement that whenever the obligation function for some node is not listed in the system description then it is implicitly understood as $\Phi(s)=\bigwedge T(s)$, with the empty conjunction being tt.

We call a PMTS positive if, for all $s \in S$, any negation occurring in $\Phi(s)$ is applied only to a parameter. A PMTS is called parameter-free if $P=\emptyset$. We can now instantiate the previously studied specification formalisms as subclasses of PMTS.

\section{Definition 2.2 A PMTS is called}

- a transition system with obligations (OTS) if it is parameter-free and positive,

- a disjunctive modal transition system (DMTS) if it is an OTS and $\Phi(s)$ is in the conjunctive normal form for all $s \in S$,

- a modal transition system (MTS) if it is a DMTS and $\Phi(s)$ is a conjunction of positive literals (transitions) for all $s \in S$, and

- an implementation (or simply a labelled transition system) if it is an MTS and $\Phi(s)=\bigwedge T(s)$ for all $s \in S$.

Note that positive PMTS, despite the absence of a general negation and the impossibility to define for example exclusive-or, can still express useful requirements like $\Phi(s)=p \Rightarrow(a, t) \wedge \neg p \Rightarrow(b, u)$ requiring in a state $s$ a conditional presence of certain transitions. Even more interestingly, we can enforce binding of actions in different states, thus ensuring certain functionality. Take a simple two state-example: $\Phi(s)=p \Rightarrow($ request,$t)$ and $\Phi(t)=p \Rightarrow$ (response, $s)$. 


\subsection{Modal Refinement}

A fundamental advantage of MTS-based formalisms is the presence of modal refinement that allows for a step-wise system design (see e.g. [4]). We shall now provide such a refinement notion for our general PMTS model so that it will specialise to the well-studied refinement notions on its subclasses. In the definition, the parameters are fixed first (persistence) followed by all valid choices modulo the fixed parameters that now behave as constants.

First we set the following notation. Let $(S, T, P, \Phi)$ be a PMTS and $\mu \subseteq P$ be a truth assignment for parameters. For $s \in S$, we denote by

$$
\operatorname{Tran}_{\mu}(s)=\{E \subseteq T(s) \mid E \cup \mu \models \Phi(s)\}
$$

the set of all admissible sets of transitions from $s$ under the fixed truth values of the parameters. For example, if $\mu=\{$ reqYfrom $R\}$ in the right-most specification in Figure 2.2 then $\operatorname{Tran}_{\mu}($ green $)=\{\{($ stop, red $)\}\}$ and $\operatorname{Tran}_{\mu}($ red $)=$ $\{\{($ ready, yellowRed $)\}\}$.

We can now define the notion of modal refinement between PMTS.

Definition 2.3 (Modal Refinement) Let $\left(S_{1}, T_{1}, P_{1}, \Phi_{1}\right)$ and $\left(S_{2}, T_{2}, P_{2}, \Phi_{2}\right)$ be two PMTSs. Let further $\mu \subseteq P_{1}$ and $\nu \subseteq P_{2}$. A binary relation $R \subseteq S_{1} \times S_{2}$ is a modal refinement respecting $\mu$ and $\nu$ if for every $(s, t) \in R$ holds

$$
\begin{aligned}
\forall M \in \operatorname{Tran}_{\mu}(s): \exists N \in \operatorname{Tran}_{\nu}(t): & \forall\left(a, s^{\prime}\right) \in M: \exists\left(a, t^{\prime}\right) \in N:\left(s^{\prime}, t^{\prime}\right) \in R \wedge \\
& \forall\left(a, t^{\prime}\right) \in N: \exists\left(a, s^{\prime}\right) \in M:\left(s^{\prime}, t^{\prime}\right) \in R .
\end{aligned}
$$

We say that $s$ modally refines $t$, denoted by $s \leq_{\mathrm{m}} t$, if for each $\mu \subseteq P_{1}$ there exists $\nu \subseteq P_{2}$ and a modal refinement $R$ respecting $\mu$ and $\nu$ such that $(s, t) \in R$.

Example 2.4 Consider the rightmost PMTS in Figure 2.2. It has two parameters req Yfrom $G$ and reqYfrom $R$ whose values can be set independently and it can be refined by the system in the middle of the figure having only one parameter req $Y$. This single parameter simply binds the two original parameters to the same value. The PMTS in the middle can be further refined into the implementations where either yellow is always used in both cases, or never at all. Notice that there are in principle infinitely many implementations of the system in the middle, however, they are all bisimilar to either of the two implementations depicted on the left of Figure 2.2

In what follows, we shall investigate the complexity of positive subclasses of PMTS. For this reason we prove the following lemma showing how the definition of modal refinement can be simplified in this particular case.

We shall first realise that in positive PMTS and for any truth assignment $\mu$, $\operatorname{Tran}_{\mu}(s)$ is upward closed, meaning that if $M \in \operatorname{Tran}_{\mu}(s)$ and $M \subseteq M^{\prime} \subseteq T(s)$ then $M^{\prime} \in \operatorname{Tran}_{\mu}(s)$.

Lemma 2.5 Consider Definition 2.3 where the right-hand side PMTS is positive. Now the condition in Definition 2.3 can be equivalently rewritten as a conjunction of conditions (2.1) and 2.2

$$
\begin{aligned}
& \forall M \in \operatorname{Tran}_{\mu}(s): \forall\left(a, s^{\prime}\right) \in M: \exists\left(a, t^{\prime}\right) \in T(t):\left(s^{\prime}, t^{\prime}\right) \in R \\
& \forall M \in \operatorname{Tran}_{\mu}(s): \operatorname{match}_{t}(M) \in \operatorname{Tran}_{\nu}(t)
\end{aligned}
$$




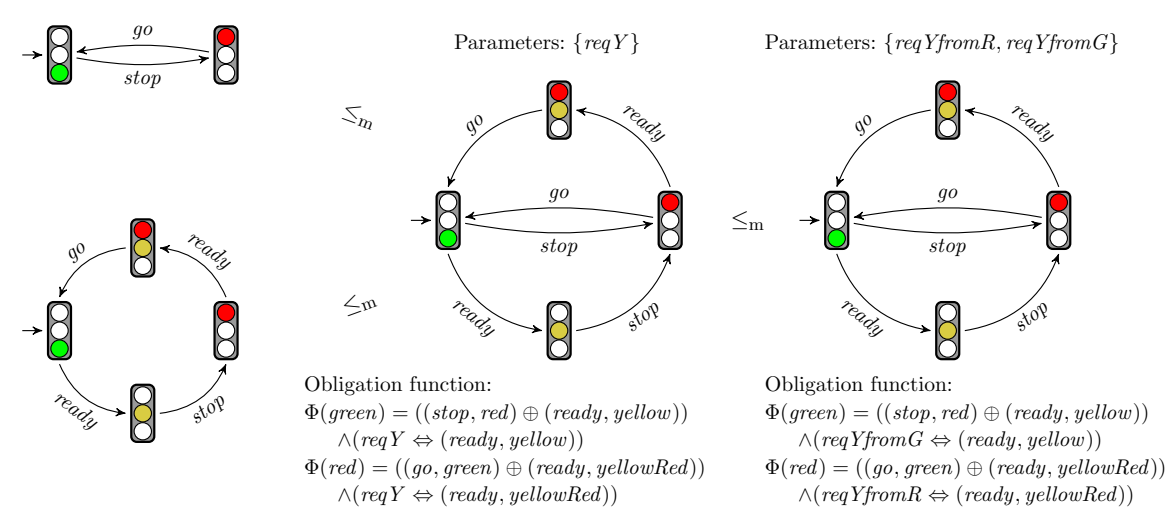

Fig. 2.2: Example of modal refinement

where $\operatorname{match}_{t}(M)$ denotes the set $\left\{\left(a, t^{\prime}\right) \in T(t) \mid \exists\left(a, s^{\prime}\right) \in M:\left(s^{\prime}, t^{\prime}\right) \in R\right\}$. If the left-hand side PMTS is moreover positive too, Condition (2.1) is equivalent to

$$
\forall\left(a, s^{\prime}\right) \in T(s): \exists\left(a, t^{\prime}\right) \in T(t):\left(s^{\prime}, t^{\prime}\right) \in R
$$

Proof We shall first argue that the condition of modal refinement is equivalent to the conjunction of Conditions (2.4) and (2.5).

$$
\begin{aligned}
& \forall M \in \operatorname{Tran}_{\mu}(s): \exists N \in \operatorname{Tran}_{\nu}(t): \forall\left(a, s^{\prime}\right) \in M: \exists\left(a, t^{\prime}\right) \in N:\left(s^{\prime}, t^{\prime}\right) \in R \\
& \forall M \in \operatorname{Tran}_{\mu}(s): \exists N \in \operatorname{Tran}_{\nu}(t): \forall\left(a, t^{\prime}\right) \in N: \exists\left(a, s^{\prime}\right) \in M:\left(s^{\prime}, t^{\prime}\right) \in R
\end{aligned}
$$

Let $\mu, \nu, R, s$ and $t$ be fixed. Definition 2.3 trivially implies both Conditions 2.4 and $(2.5)$. We now prove that 2.4 and 2.5 imply the condition in Definition 2.3

Let $M \in \operatorname{Tran}_{\mu}(s)$ be arbitrary. There is some $N_{1} \in \operatorname{Tran}_{\nu}(t)$ satisfying (2.4) and some $N_{2} \in \operatorname{Tran}_{\nu}(t)$ satisfying 2.5). Let now $N_{1}^{\prime}=\left\{\left(a, t^{\prime}\right) \in N_{1} \mid \exists\left(a, s^{\prime}\right) \in\right.$ $\left.M:\left(s^{\prime}, t^{\prime}\right) \in R\right\}$. Consider $N=N_{1}^{\prime} \cup N_{2}$. Clearly, as $\operatorname{Tran}_{\nu}(t)$ is upward closed, $N \in \operatorname{Tran}_{\nu}(t)$. Moreover, due to Condition (2.4) for every $\left(a, s^{\prime}\right) \in M$ we have some $\left(a, t^{\prime}\right) \in N_{1}$ such that $\left(s^{\prime}, t^{\prime}\right) \in R$. Clearly, $\left(a, t^{\prime}\right) \in N_{1}^{\prime}$ and thus also in $N$.

Now let $\left(a, t^{\prime}\right) \in N$ be arbitrary. If $\left(a, t^{\prime}\right) \in N_{2}$, due to Condition 2.5 we have some $\left(a, s^{\prime}\right) \in M$ such that $\left(s^{\prime}, t^{\prime}\right) \in R$. If $\left(a, t^{\prime}\right) \notin N_{2}$ then $\left(a, t^{\prime}\right) \in N_{1}^{\prime}$. The existence of $\left(a, s^{\prime}\right) \in M$ such that $\left(s^{\prime}, t^{\prime}\right) \in R$ is then guaranteed by the definition of $N_{1}^{\prime}$.

Let us now proceed with proving the claims of the lemma. Condition 2.4

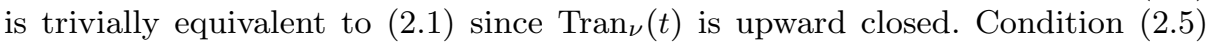
is equivalent to $(2.2$. Indeed, $(2.2)$ clearly implies $(2.5)$ and we show that also (2.5) implies 2.2). Let $M$ be arbitrary. We then have some $N$ satisfying (2.5). Clearly, $N \subseteq \operatorname{match}_{t}(M)$. Since $\operatorname{Tran}_{\nu}(t)$ is upward closed, $N \in \operatorname{Tran}_{\nu}(t) \operatorname{implies}$ $\operatorname{match}_{t}(M) \in \operatorname{Tran}_{\nu}(t)$. Due to the upward closeness of both $\operatorname{Tran}_{\mu}(s)$ and $\operatorname{Tran}_{\nu}(t)$ in the case of a positive left-hand side, the equivalence of (2.1) and (2.3) follows.

Theorem 2.6 Modal refinement as defined on PMTS coincides with the standard modal refinement notions on MTS, DMTS and OTS. On implementations it coincides with bisimulation. 
Proof The fact that Definition 2.3 coincides with modal refinement on OTS as defined in [12] is a straightforward corollary of Lemma 2.5 and its proof. Indeed, the two conditions given in 12 are exactly conditions $\sqrt{2.3)}$ and $(2.5)$. As the definition of modal refinement on OTS coincides with modal refinement on DMTS (as shown in [12]) and thus also on MTS, the proof is done.

However, for the reader's convenience, we present a direct proof that Definition 2.3 coincides with modal refinement on MTS. Assume a parameter-free PMTS $(S, T, \emptyset, \Phi)$ where $\Phi(s)$ is a conjunction of transitions for all $s \in S$, in other words it is a standard MTS where the must transitions are listed in the conjunction and the may transitions are simply present in the underlying transition system but not necessarily a part of the conjunction. Observe that every transition $(s, a, t) \in T$ is contained in some $M \in \operatorname{Tran}_{\emptyset}(s)$. Further, each must transition $(s, a, t) \in T$ is contained in all $M \in \operatorname{Tran}_{\emptyset}(s)$. Therefore, the first conjunct in Definition 2.3 requires that for all may transition from $s$ there be a corresponding one from $t$ with the successors in the refinement relation. Similarly, the second conjunct now requires that for all must transitions from $t$ there be a corresponding must transition from $s$. This is exactly the standard notion of modal refinement as introduced in [42].

\section{Thorough Refinement}

While modal refinement is defined syntactically, there is also a corresponding notion defined semantically, the so-called thorough refinement as studied e.g. in [5, 13, 16, 17. From a semantical point of view, a state $s$ of a PMTS may be seen to denote the set of its implementations, i.e.

$$
\llbracket s \rrbracket:=\left\{i \mid i \text { is an implementation and } i \leq_{\mathrm{m}} s\right\} .
$$

We can now give the definition of a thorough refinement based on the set inclusion of all possible implementations.

Definition 3.1 (Thorough Refinement) For PMTS states $s$ and $t$, we say that $s$ thoroughly refines $t$, written $s \leq_{\mathrm{t}} t$, if $\llbracket s \rrbracket \subseteq \llbracket t \rrbracket$.

We discuss the relationship of the two refinements. Firstly, the modal refinement is a sound approximation to the thorough refinement.

Proposition 3.2 Let $s$ and $t$ be PMTS states. If $s \leq_{\mathrm{m}} t$ then $s \leq_{\mathrm{t}} t$.

Proof For any $i \in \llbracket s \rrbracket$, we have $i \leq_{\mathrm{m}} s$ and due to transitivity of $\leq_{\mathrm{m}}, i \leq_{\mathrm{m}} s \leq_{\mathrm{m}} t$ implies $i \leq_{\mathrm{m}} t$, hence $i \in \llbracket t \rrbracket$.

The converse does not hold even for MTS as shown in the following classical example [18] where $s_{0} \leq_{\mathrm{t}} t_{0}$, but $s_{0} \not_{\mathrm{m}} t_{0}$.
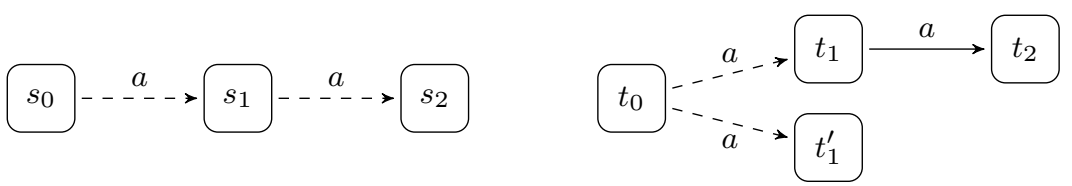
Let us now consider the subclass of deterministic PMTS. A PMTS $(S, T, P, \Phi)$ is deterministic if for every $(s, a, t),\left(s, a, t^{\prime}\right) \in T$ we have $t=t^{\prime}$. Provided that the refined MTS is deterministic, the approximation of thorough refinement via a modal refinement is now also complete [18]. This property holds even for parameter-free PMTS, as formulated in the next proposition, and it is a useful observation as deterministic systems often appear in practice [18] and checking modal refinement is - as we shall see later - computationally easier than the thorough refinement.

Proposition 3.3 Let $s_{0}$ be a PMTS state and $t_{0}$ a deterministic parameter-free PMTS state. If $s_{0} \leq_{\mathrm{t}} t_{0}$ then $s_{0} \leq_{\mathrm{m}} t_{0}$.

Proof In this proof, we use the notation $\widehat{T}(s)=\bigcup \operatorname{Tran}_{\mu}(s)$. We now fix a valuation $\mu$ of parameters and define a relation $R$ that satisfies the condition of Definition 2.3 . The relation $R$ is taken as the smallest relation such that $\left(s_{0}, t_{0}\right) \in R$ and whenever $(s, t) \in R,\left(a, s^{\prime}\right) \in \widehat{T}(s)$ and $\left(t, a, t^{\prime}\right) \in T$ then also $\left(s^{\prime}, t^{\prime}\right) \in R$. Before we prove that $R$ satisfies the conditions, we make the claim that $(s, t) \in R$ implies $s \leq_{\mathrm{t}} t$. Clearly, this holds for $\left(s_{0}, t_{0}\right)$. Suppose now that $s \leq_{\mathrm{t}} t,\left(a, s^{\prime}\right) \in \widehat{T}(s),\left(t, a, t^{\prime}\right) \in T$ and $i^{\prime}$ is an arbitrary implementation of $s^{\prime}$. Then there exists an implementation $i \in \llbracket s \rrbracket$ such that $i \stackrel{a}{\longrightarrow} i^{\prime}$. But as $s \leq_{\mathrm{t}} t, i$ is also an implementation of $t$. Therefore, as $t$ is deterministic, $i^{\prime}$ is an implementation of $t^{\prime}$, thus $s^{\prime} \leq_{\mathrm{t}} t^{\prime}$. We can now check that $R$ satisfies the condition of Definition 2.3. Let $(s, t) \in R$ and $M \in \operatorname{Tran}_{\mu}(s)$. Define $A:=\left\{a \mid \exists s^{\prime}:\left(a, s^{\prime}\right) \in M\right\}$. There is an implementation $i$ with exactly transitions under $A$. Moreover, according to the assumption it is also an implementation of $t$. Hence $N:=\left\{\left(a, t^{\prime}\right) \mid\left(t, a, t^{\prime}\right) \in T \wedge a \in A\right\}$ is an element of $\operatorname{Tran}_{\emptyset}(t)$. The two conjuncts then clearly hold by the construction of $R$.

However, the completeness fails if the refined system is deterministic but with parameters.

Example 3.4 Consider a parameter-free PMTS, in fact in this case it is sufficient to use only an MTS, $\left(\left\{s_{0}, s_{1}\right\},\left\{\left(s_{0}, a, s_{1}\right)\right\}, \emptyset,\left\{s_{0} \mapsto \mathbf{t t}, s_{1} \mapsto \mathbf{t t}\right\}\right)$ where we use the standard MTS notation, and a deterministic PMTS $\left(\left\{t_{0}, t_{1}\right\},\left\{\left(t_{0}, a, t_{1}\right)\right\},\{p\},\left\{t_{0} \mapsto\right.\right.$ $a \Leftrightarrow p, t \mapsto \mathbf{t t}\})$ below. Obviously $\llbracket s_{0} \rrbracket=\llbracket t_{0} \rrbracket$ contains the implementations with no transitions or one step $a$-transitions. Although $s_{0} \leq_{\mathrm{t}} t_{0}$, we do not have $s_{0} \leq_{\mathrm{m}} t_{0}$ as we cannot match with any valuation of $p$.
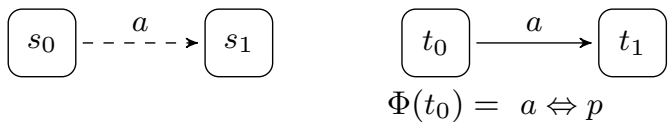

Corollary 3.5 There is a PMTS state $s$ and a deterministic PMTS state $t$ such that $s \leq_{\mathrm{t}} t$ but $s \mathbb{x}_{\mathrm{m}} t$.

In order to overcome the difficulties when modal and thorough refinement do not coincide, the underapproximation of thorough refinement by modal refinement has been complemented by an overapproximation using deterministic hull 18 and parameter-free deterministic hull 38. The property of the hull $\mathcal{D}$ is that $s \leq_{\mathrm{t}} t$ implies $s \leq_{\mathrm{m}} \mathcal{D}(t)$. The importance of using modal refinement instead of the semantically more precise thorough refinement stems from their complexities. While modal refinement on PMTS can be decided in polynomial space and for 
Table 3.1: Thorough refinement complexity and its relation to modal refinement

\begin{tabular}{cccc} 
& MTS & parameter-free PMTS & PMTS \\
\hline$\leq_{\mathrm{t} \in} \in$ & EXPTIME & NEXPTIME & 2 -EXPTIME \\
$s \leq_{\mathrm{t}} t, t$ deterministic & $\leq_{\mathrm{m}}=\leq_{\mathrm{t}}$ & $\leq_{\mathrm{m}}=\leq_{\mathrm{t}}$ & $\leq_{\mathrm{m}} \neq \leq_{\mathrm{t}}$
\end{tabular}

many subclasses even in polynomial time, deciding the thorough refinement is EXPTIME-hard already for MTS [11. The upper bounds are summarised in Table 3.1

Containment of the problem in EXPTIME for MTS was proven in 11 and for DMTS in [19] with the full proof in [8], in NEXPTIME for parameter-free PMTS in [38] with the full proof in [39]. Here we only focus on the general PMTS case. First, we show how to transform PMTS to parameter-free PMTS and DMTS and thus reduce our problems to the already solved one.

For a PMTS, we define a system where we can use any valuation of the parameters.

Definition 3.6 For a PMTS $\mathcal{M}=(S, T, P, \Phi)$ with a given state $s \in S$, we define a parameter-free PMTS called de-parameterisation $\mathcal{M}^{B}=\left(\left\{s^{B}\right\} \cup S \times 2^{P}, T^{\prime}, \emptyset, \Phi^{\prime}\right)$ with a newly added state $s^{B}$ as

- $T^{\prime}=\left\{\left(s^{B}, a,(s, \mu)\right) \mid(s, a, s) \in T, \mu \subseteq P\right\} \cup\left\{\left((s, \mu), a,\left(s^{\prime}, \mu\right) \mid\left(s, a, s^{\prime}\right) \in T\right\}\right.$,

- $\Phi^{\prime}\left(s^{B}\right)=\bigoplus_{\mu \subseteq P} \Phi(s)[\mathbf{t t} / p$ for $p \in \mu$, ff $/ p$ for $p \notin \mu,(s, \mu) / s]$, and

- $\Phi^{\prime}((s, \mu)) \stackrel{\text {. }}{=} \Phi(s)[\mathbf{t t} / p$ for $p \in \mu, \mathrm{ff} / p$ for $p \notin \mu,(s, \mu) / s]$.

The de-parameterisation is a parameter-free PMTS having exactly all the implementations of the PMTS and only one (trivial) valuation.

Proposition 3.7 Let $s$ be a PMTS state. Then $\llbracket s \rrbracket=\llbracket s^{B} \rrbracket$ and $s \leq_{\mathrm{m}} s^{B}$.

Proof For any parameter valuation $\mu$ we match it with $\emptyset$ and the modal refinement is achieved in the copy with $\mu$ fixed in the second component. Clearly, any implementation of $s^{B}$ corresponds to a particular parameter valuation and thus also to an implementation of $s$.

Remark 3.8 Notice that de-parameterisation only preserves the set of implementations but the de-parameterised system does not necessarily modally refine the original system. Moreover, the price we have to pay is a blowup exponential in $|P|$. This is, however, inevitable. Indeed, consider a PMTS $\left(\left\{s_{0}, s_{1}, s_{2}\right\},\left\{\left(s_{0}, p, s_{1}\right),\left(s_{1}, p, s_{2}\right) \mid\right.\right.$ $\left.p \in P\}, P,\left\{s_{0}, s_{1} \mapsto \bigwedge_{p \in P}(p, s) \Leftrightarrow p, s_{2} \mapsto \mathbf{t t}\right\}\right)$. Then in every equivalent parameterfree PMTS we need to remember the transitions of the first step so that we can repeat exactly these in the following step. Since there are exponentially many possibilities, the result follows.

Further, similarly to Boolean formulae with states in [9], we can transform every parameter-free PMTS to a DMTS.

Definition 3.9 For a parameter-free PMTS $\mathcal{M}=(S, T, \emptyset, \Phi)$, we define a DMTS called de-negation $\mathcal{M}^{D}=\left(S^{\prime}, T^{\prime}, \emptyset, \Phi^{\prime}\right)$ 
$-S^{\prime}=\left\{M \in \operatorname{Tran}_{\emptyset}(s) \mid s \in S\right\}$,

- $\Phi^{\prime}(M)=\bigwedge_{\left(a, s^{\prime}\right) \in M} \bigvee_{M^{\prime} \in \operatorname{Tran}_{\emptyset}\left(s^{\prime}\right)}\left(a, M^{\prime}\right)$,

and $T^{\prime}$ is minimal such that for each $M \in S^{\prime}$ and each occurrence of $\left(a, M^{\prime}\right)$ in $\Phi(M)$, we have $\left(M, a, M^{\prime}\right) \in T^{\prime}$.

However, this DMTS needs to have more initial states in order to be equivalent to the original parameter-free PMTS.

Lemma 3.10 For a state $s$ of a parameter-free PMTS, $\llbracket s \rrbracket=\bigcup_{M \in \operatorname{Tran}_{\emptyset}(s) \llbracket M \rrbracket \text { (where }}$ $M$ are taken as states of the de-negation).

Note that both transformations are exponential. The first one in $|P|$ and the second one in the branching degree. Therefore, their composition is still only singly exponential, yielding a state space where each state has two components: a valuation of original parameters and $\operatorname{Tran}_{\emptyset}$ of the original state under this valuation.

Theorem 3.11 Thorough refinement on PMTS is in 2-EXPTIME and EXPTIMEhard.

Proof Recall that thorough refinement on DMTS is in EXPTIME [5, 13. Further, note that we have reduced the PMTS and parameter-free PMTS thorough refinement problems to the one on DMTS with more initial states. However, this does not pose a problem. Indeed, let $s$ and $t$ be states of a parameter-free PMTS. We want to check whether $s \leq_{\mathrm{t}} t$. According to 8 where DMTS only have one initial state, we only need to check whether for each $M \in \operatorname{Tran}_{\emptyset}(s)$ we have $\left(M, \operatorname{Tran}_{\emptyset}(t)\right) \notin$ Avoid (defined in [8), which can clearly still be done in exponential time.

The hardness follows from the fact that thorough refinement checking on ordinary MTS is already EXPTIME-hard [13.

Due to this high complexity of the thorough refinement, we shall only deal with the modal refinement from now on. Note that the original version of modal refinement on PMTS [14] required, for $s_{0} \leq_{\mathrm{m}} t_{0}$ to hold, that there exists a fixed $R \subseteq S_{1} \times S_{2}$ such that for every $\mu \subseteq P_{1}$ there exists $\nu \subseteq P_{2}$ satisfying for each $(s, t) \in R$

$$
\begin{aligned}
\forall M \in \operatorname{Tran}_{\mu}(s): \exists N \in \operatorname{Tran}_{\nu}(t): & \forall\left(a, s^{\prime}\right) \in M: \exists\left(a, t^{\prime}\right) \in N:\left(s^{\prime}, t^{\prime}\right) \in R \wedge \\
& \forall\left(a, t^{\prime}\right) \in N: \exists\left(a, s^{\prime}\right) \in M:\left(s^{\prime}, t^{\prime}\right) \in R .
\end{aligned}
$$

Clearly, the original definition is stronger: For any two PMTS states, if $s_{0} \leq_{\mathrm{m}} t_{0}$ holds according to 14 it also holds according to Definition 2.3 Indeed, the relation for any sets of parameters can be chosen to be the fixed relation $R$. On the other hand, the opposite does not hold, as illustrated by the following example.

Example 3.12 Consider the PMTS on the left with parameter set $\{p\}$ and obligation $\Phi\left(s_{0}\right)=\left(a, s_{1}\right), \Phi\left(s_{1}\right)=\left(b, s_{2}\right) \Leftrightarrow p, \Phi\left(s_{2}\right)=\mathbf{t t}$ and the PMTS on the right with parameter set $\{q\}$ and obligation $\Phi\left(t_{0}\right)=\left(\left(a, t_{1}\right) \Leftrightarrow q\right) \wedge\left(\left(a, t_{1}^{\prime}\right) \Leftrightarrow \neg q\right), \Phi\left(t_{1}\right)=$ $\left(a, t_{2}\right), \Phi\left(t_{2}\right)=\Phi\left(t_{1}^{\prime}\right)=\mathbf{t t}$. On the one hand, according to our definition $s_{0} \leq_{\mathrm{m}} t_{0}$, we intuitively agree this should be the case (and note they also have the same set of implementations). On the other hand, the original definition does not allow to conclude modal refinement between $s_{0}$ and $t_{0}$. The reason is that depending on 
the value of $p, s_{1}$ is put in the relation either with $t_{1}$ (for $p$ being true and thus choosing $q$ true, too) or with $t_{1}^{\prime}$ (for $p$ being false and thus choosing $q$ false, too). In contrast to the original definition, our definition allows us to pick different relations for different parameter valuations.
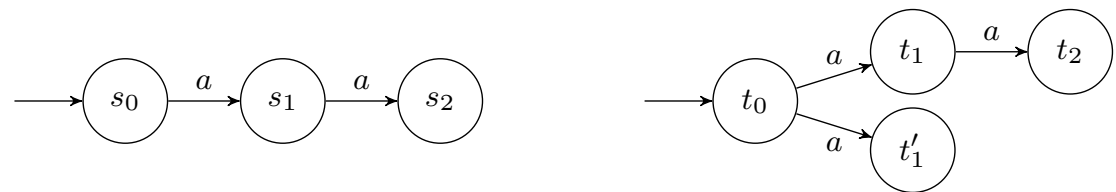

We propose our modification of the definition since it is not only more intuitive, but it also better approximates the thorough refinement and for all considered fragments of PMTS has the same complexity as the original one. Note that the two definitions coincide on parameter-free PMTS. Further, on MTS they coincide with the classical definition [42] and on labelled transition systems with bisimulation.

\section{Complexity of Modal Refinement Checking}

We shall now investigate the complexity of refinement checking on PMTS and its relevant subclasses. Without explicitly mentioning it, we assume that all considered PMTS are now finite and the decision problems are hence well defined. The complexity bounds include classes from the polynomial hierarchy (see e.g. 49]) where for example $\Sigma_{0}^{\mathrm{P}}=\Pi_{0}^{\mathrm{P}}=\mathrm{P}, \Pi_{1}^{\mathrm{P}}=\operatorname{coNP}$ and $\Sigma_{1}^{\mathrm{P}}=\mathrm{NP}$.

Let us also recall the $\mathrm{QBF}$ decision problems.

Definition 4.1 $\left(\mathrm{QBF}_{n}^{Q}\right)$ Let $A p$ be a set of atomic propositions, partitioned into $n$ sets $A p=\bigcup_{i=0}^{n} X_{i}$. Let $\phi \in \mathcal{B}(A p)$ be a Boolean formula over the set of atomic propositions $A p$ and let $Q \in\{\forall, \exists\}$ be a quantifier where by convention $\bar{\forall}=\exists$ and $\bar{\exists}=\forall$. Then a formula of the form

$$
Q X_{1} \bar{Q} X_{2} Q X_{3} \bar{Q} X_{4} \ldots \widetilde{Q} X_{n} \phi \quad \text { where } \widetilde{Q}= \begin{cases}Q & \text { if } n \text { is odd } \\ \bar{Q} & \text { if } n \text { is even }\end{cases}
$$

is an instance of $\mathrm{QBF}_{n}^{Q}$ if and only if it is true.

A formula is true means that if e.g. $Q=\exists$ then there is some partial valuation for the atomic propositions in $X_{1}$, such that for all partial valuations for the elements of $X_{2}$, there is another partial valuation for the propositions of $X_{3}$ and so on up to $X_{n}$, such that $\phi$ is satisfied by the union of all partial valuations. It is well known, see e.g. 49, that these problems are complete for the polynomial hierarchy: for each $i \geq 1, \mathrm{QBF}_{i}^{\exists}$ is $\Sigma_{i}^{\mathrm{P}}$-complete and $\mathrm{QBF}_{i}^{\forall}$ is $\Pi_{i}^{\mathrm{P}}$-complete.

\subsection{Parameter-Free Systems}

Since even the parameter-free systems have interesting expressive power and the complexity of refinement on OTS has not been studied before, we first focus on parameter-free systems. Moreover, the results of this subsection are then applied to parametric systems in the next subsection. The results are summarised in 
Table 4.1: Complexity of modal refinement checking of parameter-free systems

\begin{tabular}{|c||c|c|c|c|c|}
\hline & Boolean & Positive & pCNF & pDNF & MTS \\
\hline \hline Boolean & $\Pi_{2}^{\mathrm{P}}$-compl. & coNP-compl. & coNP-compl. & coNP-compl. & coNP-compl. \\
\hline Positive & $\Pi_{2}^{\mathrm{P}}$-compl. & coNP-compl. & P-compl. & coNP-compl. & P-compl. \\
\hline pCNF & $\Pi_{2}^{\mathrm{P}}$-compl. & coNP-compl. & P-compl. & coNP-compl. & P-compl. \\
\hline pDNF & $\Pi_{2}^{\mathrm{P}}$-compl. & P-compl. & P-compl. & P-compl. & P-compl. \\
\hline MTS & $\Pi_{2}^{\mathrm{P}}$-compl. & P-compl. & P-compl. & P-compl. & P-compl. \\
\hline Impl & NP-compl. & P-compl. & P-compl. & P-compl. & P-compl. \\
\hline
\end{tabular}

Table 4.1. The rows in the table correspond to the restrictions on the left-hand side PMTS while the columns correspond to the restrictions on the right-hand side PMTS. Boolean denotes the general system with arbitrary negation. Positive denotes the positive systems, in this case exactly OTS. We further use pCNF and pDNF to denote positive systems with formulae in conjunctive and disjunctive normal forms, respectively. In this case, pCNF coincides with DMTS. The special case of satisfaction relation, where the refining system is an implementation is denoted by Impl. We do not include Impl to the columns as it makes sense that an implementation is refined only to an implementation and here modal refinement corresponds to bisimilarity that is $\mathrm{P}$-complete [6] (see also [53]). The P-hardness is hence the obvious lower bound for all the problems mentioned in the table.

We start with the simplest NP-completeness result.

Proposition 4.2 Modal refinement between an implementation and a parameter-free PMTS is NP-complete.

Proof The containment part is straightforward. First we guess the relation $R$. As $s$ is an implementation then the set $\operatorname{Tran}_{\emptyset}(s)$ is a singleton. We thus only need to further guess $N \in \operatorname{Tran}_{\emptyset}(t)$ and then in polynomial time verify the two conjuncts in Definition 2.3

The hardness part is by a simple reduction from $\operatorname{SAT}$. Let $\varphi\left(x_{1}, \ldots, x_{n}\right)$ be a given Boolean formula (instance of SAT). We construct two parameter-free PMTSs $(S, T, \emptyset, \Phi)$ and $\left(S^{\prime}, T^{\prime}, \emptyset, \Phi^{\prime}\right)$ such that (i) $S=\left\{s, s^{\prime}\right\}, T=\left(s, a, s^{\prime}\right), \Phi(s)=$ $\left(a, s^{\prime}\right)$ and $\Phi\left(s^{\prime}\right)=\mathbf{t t}$ and (ii) $S^{\prime}=\left\{t, t_{1}, \ldots, t_{n}\right\}, T=\left\{\left(t, a, t_{i}\right) \mid 1 \leq i \leq n.\right\}$, $\Phi(t)=\varphi\left[\left(a, t_{i}\right) / x_{i}\right]$ and $\Phi\left(t_{i}\right)=\mathbf{t t}$ for all $i, 1 \leq i \leq n$. Clearly, $\varphi$ is satisfiable if and only if $s \leq_{\mathrm{m}} t$.

Next we show that modal refinement is $\Pi_{2}^{\mathrm{P}}$-complete. The following proposition introduces a gadget used also later on in other hardness results. We will refer to it as the $*$-construction.

Proposition 4.3 Modal refinement between two parameter-free PMTS is $\Pi_{2}^{\mathrm{P}}$-hard even if the left-hand side is an MTS.

Proof The proof is by polynomial time reduction from the validity of the quantified Boolean formula $\psi \equiv \forall x_{1} \ldots \forall x_{n} \exists y_{1} \ldots \exists y_{m}: \varphi\left(x_{1}, \ldots, x_{n}, y_{1}, \ldots, y_{m}\right)$ to the refinement checking problem $s \leq_{\mathrm{m}} t$ where $s$ and $t$ are given as follows. 

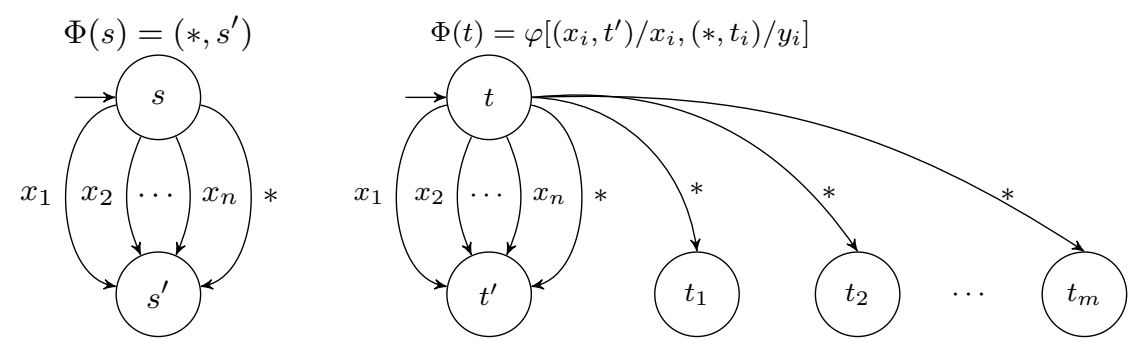

Assume that $\psi$ is true. Let $M \in \operatorname{Tran}_{\emptyset}(s)$ (clearly $\left(*, s^{\prime}\right) \in M$ ) and we want to argue that there is $N \in \operatorname{Tran}_{\emptyset}(t)$ with $\left(*, t^{\prime}\right) \in N$ such that for all $\left(x_{i}, s^{\prime}\right) \in M$ there is $\left(x_{i}, t^{\prime}\right) \in N$ (clearly the states $s^{\prime}, t^{\prime}$ and $t_{i}$ are in modal refinement) and for all $\left(x_{i}, t^{\prime}\right) \in N$ there is $\left(x_{i}, s^{\prime}\right) \in M$. Such an $N$ can be found by simply including $\left(x_{i}, t^{\prime}\right)$ whenever $\left(x_{i}, s^{\prime}\right) \in M$ and by adding also $\left(*, t^{\prime}\right)$ into $N$. As $\psi$ is true, we include into $N$ also all $\left(*, t_{i}\right)$ whenever $y_{i}$ is set to true in $\psi$. Hence we get $s \leq_{\mathrm{m}} t$.

On the other hand if $\psi$ is false then we pick $M \in \operatorname{Tran}_{\emptyset}(s)$ such that $M$ corresponds to the values of $x_{i}$ 's such that there are no values of $y_{1}, \ldots, y_{m}$ that make $\psi$ true. This means that there is no $N \in \operatorname{Tran}_{\emptyset}(t)$ that would contain exactly those $\left(x_{i}, t^{\prime}\right)$ that correspond to $\left(x_{i}, s^{\prime}\right)$ in $M$. Hence, $s \not \underline{\mathrm{m}}_{\mathrm{m}} t$.

Proposition 4.4 Modal refinement between two parameter-free PMTS is in $\Pi_{2}^{\mathrm{P}}$.

Proof We first note that the condition of Definition 2.3 can itself be verified in $\Pi_{2}^{\mathrm{P}}$. Indeed, if we universally guess $M$ and then existentially guess $N$, the rest of the condition is verifiable in polynomial time.

We could therefore, in principle, guess the relation $R$ and then verify that the condition holds for all pairs $(s, t) \in R$. However, this would increase the complexity bound to $\Sigma_{3}^{\mathrm{P}}$. Instead, we initially include all (polynomially many) pairs into $R$ and for each pair check the condition. If it fails, we remove the pair and continue until we reach the greatest fixed point. This standard method is usually known as the coinductive computation of $R$.

However, a naive implementation of this idea would only prove that modal refinement is in $\Delta_{3}^{\mathrm{P}}$ as we have a polynomial algorithm with $\Pi_{2}^{\mathrm{P}}$ oracle. To actually show the statement of the proposition, we describe a coNP ${ }^{N P}$ algorithm, i.e. an algorithm with universal branching that uses an NP oracle.

Suppose that we want to check if $s \leq_{\mathrm{m}} t$. The algorithm works as follows:

1. Set $R:=S \times S^{\prime}$ (all pairs of states).

2. If $R$ does not contain $(s, t)$, reject.

3. Universally choose $\left(s^{\prime}, t^{\prime}\right) \in R$ and $M \in \operatorname{Tran}\left(s^{\prime}\right)$.

4. Using the NP-oracle, decide whether there exists $N \in \operatorname{Tran}\left(t^{\prime}\right)$ such that the refinement condition for $\left(s^{\prime}, t^{\prime}\right)$ is satisfied.

5. If the result was false, remove $\left(s^{\prime}, t^{\prime}\right)$ from $R$ and go back to step 2 Otherwise, accept.

It may be clearly seen that the algorithm accepts iff $s \leq_{\mathrm{m}} t$.

\subsubsection{Positive Right-Hand Side.}

We have now solved all the cases where the right-hand side is arbitrary. We now look at the cases where the right-hand side is positive. In the proofs that follow 
we shall use the alternative characterisation of refinement from Lemma 2.5. The following proposition determines the subclasses on which modal refinement can be decided in polynomial time.

Proposition 4.5 Modal refinement on parameter-free PMTS is in P, provided that both sides are positive and either the left-hand side is in $p D N F$ or the right-hand side is in $p C N F$.

Proof Due to Lemma 2.5 the refinement is equivalent to the conjunction of $(2.3)$ and $(2.2)$. Clearly, 2.3 can be checked in P. We show that Condition 2.2 can be verified in $\mathrm{P}$ too. Recall that 2.2 says that

$$
\forall M \in \operatorname{Tran}_{\mu}(s): \operatorname{match}_{t}(M) \in \operatorname{Tran}_{\nu}(t),
$$

where $\operatorname{match}_{t}(M)=\left\{\left(a, t^{\prime}\right) \in T(t) \mid \exists\left(a, s^{\prime}\right) \in M:\left(s^{\prime}, t^{\prime}\right) \in R\right\}$

First assume that the left-hand side is in pDNF. If for some $M$ Condition $(2.2)$ is satisfied then it is also satisfied for all $M^{\prime} \supseteq M$, as $\operatorname{Tran}_{\mu}(s)$ is upwards closed. It it thus sufficient to verify the condition for all minimal elements (wrt. inclusion) of $\operatorname{Tran}_{\mu}(s)$. In this case these correspond to the clauses of $\Phi(s)$. Thus we get a polynomial time algorithm as shown in Algorithm 1 .

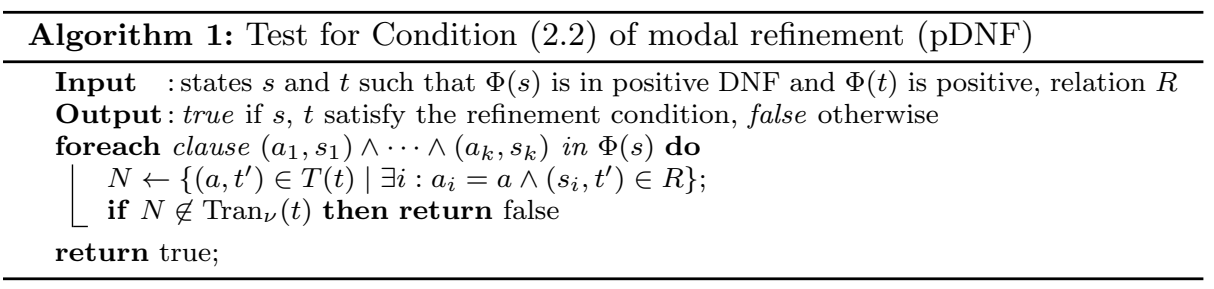

Second, assume that the right-hand side is in pCNF. Note that Condition 2.2 can be equivalently stated as

$$
\forall M: \operatorname{match}_{t}(M) \notin \operatorname{Tran}_{\nu}(t) \Rightarrow M \notin \operatorname{Tran}_{\mu}(s) .
$$

As $\Phi(t)$ is in conjunctive normal form then $N \in \operatorname{Tran}_{\nu}(t)$ is equivalent to saying that $N$ has nonempty intersection with each clause of $\Phi(t)$. We may thus enumerate all maximal $N \notin \operatorname{Tran}_{\nu}(t)$. Having a maximal $N \notin \operatorname{Tran}_{\nu}(t)$, we can easily construct $M$ such that $N=\operatorname{match}_{t}(M)$. This leads to the polynomial time Algorithm 2

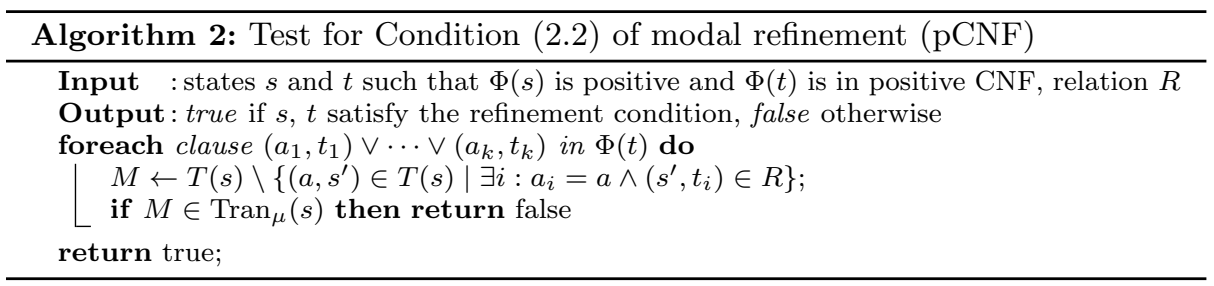

The statement of the proposition thus follows. 
Proposition 4.6 Modal refinement on parameter-free PMTS is in coNP, if the righthand side is positive.

Proof Due to Lemma 2.5 we can solve the two refinement conditions separately. Furthermore, both Condition (2.1) and 2.2 of Lemma 2.5 can be checked in coNP. The computation of $R$ is then done similarly to the algorithm described in the proof of Proposition 4.4

Proposition 4.7 Modal refinement on parameter-free systems is coNP-hard, even if the left-hand side is in positive CNF and the right-hand side is in positive DNF.

Proof We reduce SAT into non-refinement. Let $\varphi\left(x_{1}, \ldots, x_{n}\right)$ be a formula in CNF. We modify $\varphi$ into an equivalent formula $\varphi^{\prime}$ as follows: add new variables $\tilde{x}_{1}, \ldots$, $\tilde{x}_{n}$, change all occurrences of $\neg x_{i}$ into $\tilde{x}_{i}$ for all $i$, and add new clauses $\left(x_{i} \vee \tilde{x}_{i}\right)$ and $\left(\neg x_{i} \vee \neg \tilde{x}_{i}\right)$.

Observe now that all clauses contain either all positive literals or all negative literals. Let $\psi^{+}$denote a CNF formula that contains all positive clauses of $\varphi^{\prime}$ and $\psi^{-}$denote a CNF formula that contains all negative clauses of $\varphi^{\prime}$. As $\varphi^{\prime}=\psi^{+} \wedge \psi^{-}$ it is clear that $\varphi^{\prime}$ is satisfiable if and only if $\left(\psi^{+} \Rightarrow \neg \psi^{-}\right)$is not valid.

We now construct two parameter-free PMTSs $(S, T, \emptyset, \Phi)$ and $\left(S^{\prime}, T^{\prime}, \emptyset, \Phi^{\prime}\right)$ over $\Sigma=\left\{x_{1}, \ldots, x_{n}, \tilde{x}_{1}, \ldots, \tilde{x}_{n}\right\}$ as follows: (i) $S=\left\{s, s^{\prime}\right\}, T=\left\{\left(s, x_{i}, s^{\prime}\right),\left(s, \tilde{x}_{i}, s^{\prime}\right) \mid 1 \leq\right.$ $i \leq n\}, \Phi(s)=\psi^{+}\left[\left(x_{i}, s^{\prime}\right) / x_{i},\left(\tilde{x}_{i}, s^{\prime}\right) / \tilde{x}_{i}\right]$ and $\Phi\left(s^{\prime}\right)=\mathbf{t t}$, and (ii) $S^{\prime}=\left\{t, t^{\prime}\right\}, T^{\prime}=$ $\left\{\left(t, x_{i}, t^{\prime}\right),\left(t, \tilde{x}_{i}, t\right) \mid 1 \leq i \leq n\right\}, \Phi(t)=\neg \psi^{-}\left[\left(x_{i}, t^{\prime}\right) / x_{i},\left(\tilde{x}_{i}, t^{\prime}\right) / \tilde{x}_{i}\right]$ and $\Phi\left(t^{\prime}\right)=\mathbf{t t}$. Note that by pushing the negation of $\psi^{-}$inside, the formula $\Phi(t)$ can be written as pDNF. It is easy to see that now $s \leq_{\mathrm{m}} t$ if and only if $\left(\psi^{+} \Rightarrow \neg \psi^{-}\right)$is valid. Therefore, $s \underline{\mathrm{m}}_{\mathrm{m}} t$ if and only if $\varphi$ is satisfiable.

Proposition 4.8 Modal refinement on parameter-free PMTS is coNP-hard, even if the right-hand side is an MTS.

Proof We prove the hardness by a reduction from SAT to non-refinement. Let $\varphi\left(x_{1}, \ldots, x_{n}\right)$ be a Boolean formula (instance of SAT). We construct two parameterfree PMTSs $(S, T, \emptyset, \Phi)$ and $\left(S^{\prime}, T^{\prime}, \emptyset, \Phi^{\prime}\right)$ over $\Sigma=\{a, b\}$ as follows:

1. $S=\left\{s, s_{1}, \ldots, s_{n}\right\}, T=\left\{\left(s, a, s_{i}\right) \mid 1 \leq i \leq n\right\}, \Phi(s)=\varphi\left[\left(a, s_{i}\right) / x_{i}\right], \Phi\left(s_{i}\right)=\mathbf{t t}$ for all $i$.

2. $S^{\prime}=\{t\}, T^{\prime}=\{(t, b, t)\}, \Phi^{\prime}(t)=(b, t)$. (Clearly, this is an MTS.)

Let now $\varphi$ be satisfiable and let $M \in \operatorname{Tran}_{\emptyset}(s)$ be arbitrary. Clearly, there can be no match $N \in \operatorname{Tran}_{\emptyset}(t)$ and thus $s \underline{\mathrm{m}}_{\mathrm{m}} t$.

Let now $\varphi$ be unsatisfiable. This means that $\operatorname{Tran}_{\emptyset}(s)=\emptyset$ and the refinement condition trivially holds. Thus $s \leq_{\mathrm{m}} t$.

\subsection{Systems with Parameters}

In the sequel we investigate the complexity of refinement checking in the general case of PMTS with parameters. The complexities are summarised in Table 4.2 We start with an observation of how the results on parameter-free systems can be applied to the parametric case. 
Table 4.2: Complexity of modal refinement checking with parameters

\begin{tabular}{|c||c|c|c|c|}
\hline & Boolean & positive & pCNF & pDNF \\
\hline \hline Boolean & $\Pi_{4}^{\mathrm{P}}$-complete & $\Pi_{3}^{\mathrm{P}}$-complete & $\Pi_{3}^{\mathrm{P}}$-complete & $\Pi_{3}^{\mathrm{P}}$-complete \\
\hline positive & $\Pi_{4}^{\mathrm{P}}$-complete & $\Pi_{3}^{\mathrm{P}}$-complete & $\Pi_{2}^{\mathrm{P}}$-complete & $\Pi_{3}^{\mathrm{P}}$-complete \\
\hline pCNF & $\Pi_{4}^{\mathrm{P}}$-complete & $\Pi_{3}^{\mathrm{P}}$-complete & $\Pi_{2}^{\mathrm{P}}$-complete & $\Pi_{3}^{\mathrm{P}}$-complete \\
\hline pDNF & $\Pi_{4}^{\mathrm{P}}$-complete & $\Pi_{2}^{\mathrm{P}}$-complete & $\Pi_{2}^{\mathrm{P}}$-complete & $\Pi_{2}^{\mathrm{P}}$-complete \\
\hline MTS & $\Sigma_{3}^{\mathrm{P}}$-complete & NP-complete & NP-complete & NP-complete \\
\hline Impl & $\mathrm{NP}$-complete & NP-complete & NP-complete & NP-complete \\
\hline
\end{tabular}

Proposition 4.9 The complexity upper bounds from Table 4.1 carry over to Table 4.2. as follows. If the modal refinement in the parameter-free case is in NP, coNP or $\Pi_{2}^{\mathrm{P}}$, then the modal refinement with parameters is in $\Pi_{2}^{\mathrm{P}}, \Pi_{3}^{\mathrm{P}}$ and $\Pi_{4}^{\mathrm{P}}$, respectively. Moreover, if the left-hand side is an MTS, the complexity upper bounds shift from NP and $\Pi_{2}^{\mathrm{P}}$ to $\mathrm{NP}$ and $\Sigma_{3}^{\mathrm{P}}$, respectively.

Proof In the first case, we first universally choose $\mu$, we then existentially choose $\nu$ and modify the formulae $\Phi(s)$ and $\Phi(t)$ by evaluating the parameters. This does not change the normal form/positiveness of the formulae. We then perform the algorithm for the parameter-free refinement. For the second case note that implementations and MTS have no parameters and we may simply choose (existentially) $\nu$ and run the algorithm for the parameter-free refinement.

We now focus on the respective lower bounds.

Proposition 4.10 Modal refinement between an implementation and a right-hand side in positive CNF or in positive DNF is NP-hard.

Proof The proof is by reduction from SAT. Let $\varphi\left(x_{1}, \ldots, x_{n}\right)$ be a formula in CNF and let $\varphi_{1}, \varphi_{2}, \ldots, \varphi_{k}$ be the clauses of $\varphi$. We construct two PMTSs $(S, T, P, \Phi)$ and $\left(S^{\prime}, T^{\prime}, P^{\prime}, \Phi^{\prime}\right)$ over the action alphabet $\Sigma=\left\{a_{1}, \ldots, a_{k}\right\}$ as follows: (i) $S=\left\{s, s^{\prime}\right\}$, $T=\left\{\left(s, a_{i}, s^{\prime}\right) \mid 1 \leq i \leq k\right\}, P=\emptyset, \Phi(s)=\bigwedge_{1 \leq i \leq k}\left(a_{i}, s^{\prime}\right)$ and $\Phi\left(s^{\prime}\right)=\mathbf{t t}$ and (ii) $S^{\prime}=\{t\} \cup\left\{t_{i} \mid 1 \leq i \leq k\right\}, T^{\prime}=\left\{\left(t, a_{i}, t_{i}\right) \mid 1 \leq i \leq k\right\}, P^{\prime}=\left\{x_{1}, \ldots, x_{n}\right\}$, $\Phi^{\prime}(t)=\bigwedge_{1 \leq i \leq k}\left(a_{i}, t_{i}\right)$ and $\Phi^{\prime}\left(t_{i}\right)=\varphi_{i}$ for all $1 \leq i \leq k$. Notice that each $\varphi_{i}$ in $\Phi^{\prime}\left(t_{i}\right)$ is in positive form as we negate only the parameters $x_{i}$ and every clause $\varphi_{i}$ is trivially in DNF. Now we easily get that $s \leq_{\mathrm{m}} t$ if and only if $\varphi$ is satisfiable.

Proposition 4.11 Modal refinement is $\Sigma_{3}^{\mathrm{P}}$-hard even if the left-hand side is an MTS.

Proof The proof is done using the construction of the proof of Proposition 4.3 with parameters added on the right-hand side.

We will make a reduction from the validity of the quantified Boolean formula $\psi \equiv \exists z_{1}, \ldots, z_{k} \forall x_{1} \ldots \forall x_{n} \exists y_{1} \ldots \exists y_{m}: \varphi\left(z_{1}, \ldots, z_{k}, x_{1}, \ldots, x_{n}, y_{1}, \ldots, y_{m}\right)$ to the refinement checking problem $s \leq_{\mathrm{m}} t$ where $s$ and $t$ are given as follows. Moreover, the right-hand side system has $\left\{z_{1}, \ldots, z_{k}\right\}$ as its set of parameters. 

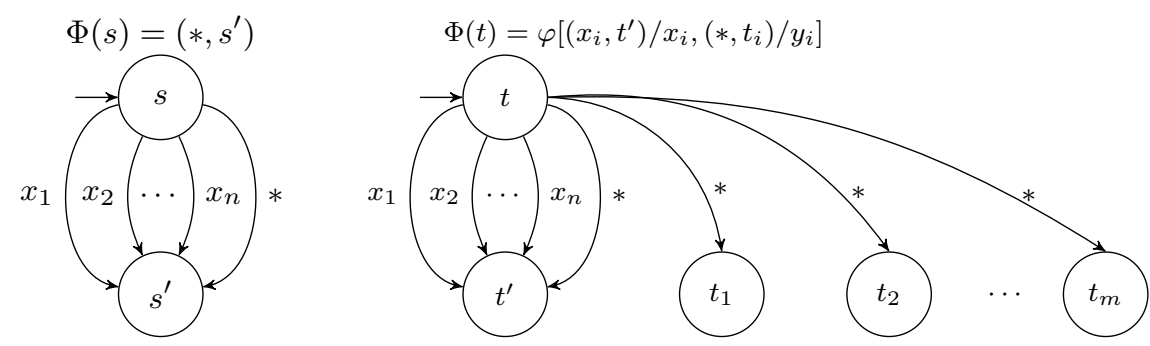

Assume that $\psi$ is true. Then there exists a valuation $\nu$ on $\left\{z_{1}, \ldots, z_{k}\right\}$ such that $\forall x: \exists y: \varphi(x, y)$ is true. Using now the same argument as that in the proof of Proposition 4.3, we get that $s \leq_{\mathrm{m}} t$.

On the other hand assume that $\psi$ is false and let $\nu$ be an arbitrary valuation on $\left\{z_{1}, \ldots, z_{k}\right\}$. We then may again use the reasoning in the proof of Proposition 4.3 to get that $s \not \underline{\mathrm{m}}_{\mathrm{m}} t$.

The following proof introduces a gadget used also later on in other hardness results. We refer to it as CNF-binding. Further, we use the $*$-construction here.

Proposition 4.12 Modal refinement is $\Pi_{4}^{\mathrm{P}}$-hard even if the left-hand side is in positive CNF.

Proof Consider a $\mathrm{QBF}_{4}^{\forall}$ instance, a formula $\psi=\forall x \exists y \forall z \exists w: \varphi(x, y, z, w)$ with $\varphi$ in CNF and $x, y, z, w$ vectors of length $n$. We construct two systems $s$ and $t$ and use the variables $\left\{x_{1}, \ldots, x_{n}\right\}$ as parameters for the left-hand side system $s$, and $\left\{y_{1}, \ldots, y_{n}\right\}$ as parameters for the right-hand side system $t$.

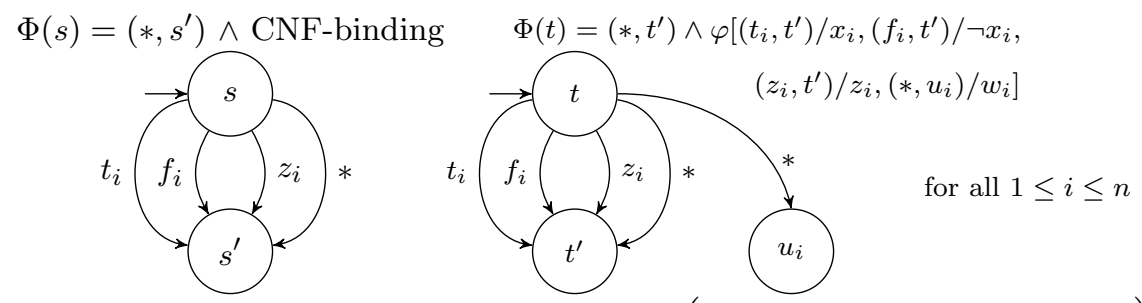

On the left we require $\Phi(s)=\left(*, s^{\prime}\right) \wedge \bigwedge_{1 \leq i \leq n}\left(\left(x_{i} \Rightarrow\left(t_{i}, s^{\prime}\right)\right) \wedge\left(\neg x_{i} \Rightarrow\left(f_{i}, s^{\prime}\right)\right)\right.$ and call the latter conjunct $C N F$-binding. Thus the value of each parameter $x_{i}$ is "saved" into transitions of the system. Note that although both $t_{i}$ and $f_{i}$ may be present, a "minimal" implementation contains exactly one of them. On the right-hand side the transitions look similar but we require $\Phi(t)=(*, t) \wedge \varphi^{\prime}$ where $\varphi^{\prime}$ is created from $\varphi$ by changing every positive literal $x_{i}$ into $\left(t_{i}, t^{\prime}\right)$, every negative literal $\neg x_{i}$ into $\left(f_{i}, t^{\prime}\right)$, every $z_{i}$ into $\left(z_{i}, t^{\prime}\right)$, and every $w_{i}$ into $\left(*, u_{i}\right)$.

We show that $\psi$ is true iff $s \leq_{\mathrm{m}} t$. Assume first that $\psi$ is true. Therefore, for every choice of parameters $x_{i}$ there is a choice of parameters $y_{i}$ so that $\forall z \exists w: \varphi(x, y, z, w)$ is true and, moreover, $t_{i}$ or $f_{i}$ is present on the left whenever $x_{i}$ or $\neg x_{i}$ is true, respectively (and possibly even if it is false). We set exactly all these transitions $t_{i}$ and $f_{i}$ on the right, too. Further, for every choice of transitions $z_{i}$ on the left there are $w_{i}$ 's so that $\varphi(x, y, z, w)$ holds. On the right, we implement a transition $\left(z_{i}, t^{\prime}\right)$ for each $z_{i}$ set to true and $\left(*, u_{i}\right)$ for each $w_{i}$ set to true. Now $\varphi^{\prime}$ is satisfied as it has only positive occurrences of $\left(t_{i}, t^{\prime}\right)$ and $\left(f_{i}, t^{\prime}\right)$ and hence the extra $t_{i}$ 's and $f_{i}$ 's do not matter. Now for every implementation of $s$ we obtained an implementation 
of $t$. Moreover, their transitions match. Indeed, $t_{i}$ 's and $f_{i}$ 's were set the same as on the left, similarly for $z_{i}$ 's. As for the $*$-transition, we use the same argumentation as in the original $*$-construction. On the left, there is always one. On the right, there can be more of them due to $w_{i}$ 's but at least one is also guaranteed by $\Phi(t)$.

Let now $s \leq_{\mathrm{m}} t$. Then for every choice of $x_{i}$ 's - and thus also for every choice of exactly one transition of $t_{i}, f_{i}$ for each $i$ - there are $y_{i}$ 's so that every choice of transitions $z_{i}$ can be matched on the right so that $\varphi^{\prime}$ is true with some transitions $\left(*, u_{i}\right)$. Since choices of $t_{i} / f_{i}$ correspond exactly to choices of $x_{i}$ it only remains to set $w_{i}$ true for each transition $\left(*, u_{i}\right)$ on the right, thus making $\varphi$ true.

Based on the idea of CNF-binding, we also prove the following two propositions.

Proposition 4.13 Modal refinement is $\Pi_{2}^{\mathrm{P}}$-hard even if both sides are in positive CNF.

Proof Recall that positive means that there may be negations, but only limited to parameter literals. The proof is done by reduction from the validity of $\forall x_{1}, \ldots, x_{n} \exists y_{1}, \ldots, y_{m}: \varphi\left(x_{1}, \ldots, x_{n}, y_{1}, \ldots, y_{m}\right)$, where $\varphi$ is in CNF. The idea is that the left-hand side has only $x_{i}$ as parameters while the right-hand side has $y_{i}$ as parameters. To ensure that the valuation of $x_{i}$ is the same on both sides, we bind them through transitions.

Let $\Sigma=\left\{t_{1}, \ldots, t_{n}, f_{1}, \ldots, f_{n}\right\}$ be the set of actions. The systems $(S, T, P, \Phi)$ and $\left(S^{\prime}, T^{\prime}, P^{\prime}, \Phi^{\prime}\right)$ are built as follows: $S=\left\{s, s^{\prime}\right\}, T=\left\{\left(s, t_{i}, s^{\prime}\right),\left(s, f_{i}, s^{\prime}\right) \mid 1 \leq i \leq n\right\}$, $P=\left\{x_{1}, \ldots, x_{n}\right\}, \Phi(s)=\bigwedge_{1 \leq i \leq n}\left(\left(x_{i} \Rightarrow\left(t_{i}, s^{\prime}\right)\right) \wedge\left(\neg x_{i} \Rightarrow\left(f_{i}, s^{\prime}\right)\right)\right.$ (note that this may be written in positive $\mathrm{CNF}), \Phi\left(s^{\prime}\right)=\mathbf{t t} ; S^{\prime}=\left\{t, t^{\prime}\right\}, T^{\prime}=\left\{\left(t, t_{i}, t^{\prime}\right),\left(t, f_{i}, t^{\prime}\right) \mid\right.$ $1 \leq i \leq n\}, P^{\prime}=\left\{y_{1}, \ldots, y_{m}\right\}, \Phi^{\prime}(t)=\varphi\left[\left(t_{i}, t^{\prime}\right) / x_{i},\left(f_{i}, t^{\prime}\right) / \neg x_{i}\right], \Phi^{\prime}\left(t^{\prime}\right)=\mathbf{t t}$. We now claim that $\forall x \exists y: \varphi$ holds if and only if $s \leq_{\mathrm{m}} t$. We show the two implications separately.

Let first $\forall x \exists y: \varphi$ hold. Let $\mu \subseteq P$ be arbitrary. As this is a truth valuation on the $x_{i}$ variables, we know that there exists a valuation on the $y_{i}$ variables such that $\varphi$ holds. Let $\nu \subseteq P^{\prime}$ be such a valuation. Let further $M \in \operatorname{Tran}_{\mu}(s)$ be arbitrary. Clearly, if $x_{i} \in \mu$ then $\left(t_{i}, s^{\prime}\right) \in M$ and if $x_{i} \notin \mu$ then $\left(f_{i}, s^{\prime}\right) \in M$.

We set $N=\left\{\left(x, t^{\prime}\right) \mid\left(x, s^{\prime}\right) \in M\right\}$. Clearly, such $N$ satisfies both conjuncts of the refinement definition. We need to show that $N \in \operatorname{Tran}_{\nu}(t)$. We thus need to show that $N$ satisfies all the clauses in $\Phi^{\prime}(t)=\varphi\left[\left(t_{i}, t^{\prime}\right) / x_{i},\left(f_{i}, t^{\prime}\right) / \neg x_{i}\right]$.

We use the fact that $\varphi$ holds, given the current valuation $\mu$ on $x_{i}$ and $\nu$ on $y_{i}$. Let $\left(\ell_{1} \vee \ell_{2} \vee \cdots \vee \ell_{k}\right)$ be an arbitrary clause of $\varphi$. Clearly, at least one literal was satisfied. If that literal was $y_{i}$ or $\neg y_{i}$ then the same literal appears in the modified clause of $\Phi^{\prime}(t)$ and we are done. If that literal was $x_{i}$ then it has been changed into $\left(t_{i}, t^{\prime}\right)$, but as $x_{i} \in \mu$, we have that $\left(t_{i}, t^{\prime}\right) \in N$. Similarly, if that literal was $\neg x_{i}$ then it has been changed into $\left(f_{i}, t^{\prime}\right)$, but as $x_{i} \notin \mu$, we have that $\left(f_{i}, t^{\prime}\right) \in N$. Thus $s \leq_{\mathrm{m}} t$.

For the other implication let $\exists x \forall y: \neg \varphi$ hold. We show that $s \not \mathbb{\mathrm { m }}_{\mathrm{m}} t$. Let $\mu$ be the valuation of $x_{i}$ such that $\exists x \forall y: \neg \varphi$ holds. Let $\nu$ be arbitrary. This corresponds to a valuation on $y_{i}$.

We now set $M=\left\{\left(t_{i}, s^{\prime}\right) \mid x_{i} \in \mu\right\} \cup\left\{\left(f_{i}, s^{\prime}\right) \mid x_{i} \notin \mu\right\}$. Clearly, $M \in \operatorname{Tran}_{\mu}(s)$. Let further $N \in \operatorname{Tran}_{\nu}(t)$. (If $\operatorname{Tran}_{\nu}(t)=\emptyset$, we are done.)

We know that given the current $x$ and $y$ valuation, $\varphi$ does not hold. This means that there exists at least one clause of $\varphi$ that is false. Let $\left(\ell_{1} \vee \ell_{2} \vee \cdots \vee \ell_{k}\right)$ be such clause. All $\ell_{j}$ are false, given current valuation $\mu$ and $\nu$. However, the modified 
clause of $\Phi^{\prime}(t)$ corresponding to this one is satisfied by $N$ (valuation of $\left(t_{i}, t^{\prime}\right)$ and $\left.\left(f_{i}, f^{\prime}\right)\right)$ as $N \in \operatorname{Tran}_{\nu}(t)$.

Therefore, for some $i$, either $\left(t_{i}, t^{\prime}\right) \in N$ while $x_{i} \notin \mu$ or $\left(f_{i}, t^{\prime}\right) \in N$ while $x_{i} \in \mu$. In both cases $N$ does not satisfy the second conjunct part of the modal refinement definition. Therefore $s \not \underline{\mathrm{m}}_{\mathrm{m}} t$.

The next proposition again reuses the idea of CNF-binding in the very same fashion as above. Moreover, it handles more actions, more precisely those that appear as $z_{i}$ 's in Proposition 4.12. Thus, the proof is the same, omitting the *-construction. Therefore, we only provide the reduction without repeating the formal arguments that it indeed works.

Proposition 4.14 Modal refinement is $\Pi_{3}^{\mathrm{P}}$-hard for the left-hand side in positive CNF and the right-hand side in positive DNF.

Proof The proof is done by reduction from the validity of the quantified Boolean formula $\forall x_{1}, \ldots, x_{k} \exists y_{1}, \ldots, y_{l} \forall z_{1}, \ldots, z_{m}: \varphi$ with $\varphi$ in DNF.

Let the action alphabet be $\Sigma=\left\{t_{1}, \ldots, t_{k}, f_{1}, \ldots, f_{k}, z_{1}, \ldots, z_{m}\right\}$.

The two systems $(S, T, P, \Phi)$ and $\left(S^{\prime}, T^{\prime}, P^{\prime}, \Phi^{\prime}\right)$ are built as follows: $S=\left\{s, s^{\prime}\right\}$, $T=\left\{\left(s, t_{i}, s^{\prime}\right),\left(s, f_{i}, s^{\prime}\right) \mid 1 \leq i \leq k\right\} \cup\left\{\left(s, z_{j}, s^{\prime}\right) \mid 1 \leq j \leq m\right\}, P=\left\{x_{1}, \ldots, x_{k}\right\}$, $\Phi(s)=\bigwedge_{1 \leq i \leq n}\left(\left(x_{i} \Rightarrow\left(t_{i}, s^{\prime}\right)\right) \wedge\left(\neg x_{i} \Rightarrow\left(f_{i}, s^{\prime}\right)\right), \Phi\left(s^{\prime}\right)=\mathbf{t t} ; S^{\prime}=\left\{t, t^{\prime}\right\}, T^{\prime}=\right.$ $\left\{\left(t, t_{i}, t^{\prime}\right),\left(t, f_{i}, t^{\prime}\right) \mid 1 \leq i \leq k\right\} \cup\left\{\left(t, z_{j}, t^{\prime}\right) \mid 1 \leq j \leq m\right\}, P^{\prime}=\left\{y_{1}, \ldots, y_{k}\right\}, \Phi^{\prime}(t)=$ $\varphi\left[\left(t_{i}, t^{\prime}\right) / x_{i},\left(f_{i}, t^{\prime}\right) / \neg x_{i},\left(z_{i}, t^{\prime}\right) / z_{i}\right], \Phi^{\prime}\left(t^{\prime}\right)=\mathbf{t t}$.

Now $\forall x \exists y \forall z: \varphi(x, y, z)$ holds if and only if $s \leq_{\mathrm{m}} t$.

The following three propositions use a modification of the CNF-binding idea called DNF-binding. Instead of $\left(x_{i} \Rightarrow\left(t_{i}, s^{\prime}\right)\right) \wedge\left(\neg x_{i} \Rightarrow\left(f_{i}, s^{\prime}\right)\right)$ we use $\left(x_{i} \wedge\left(t_{i}, s^{\prime}\right)\right) \vee$ $\left(\neg x_{i} \wedge\left(f_{i}, s^{\prime}\right)\right)$ to bind parameters of the left-hand side system with transitions of the right-hand side system. The binding works slightly differently, as with DNF we are unable to make a conjunction of such formulae for all $i$. We thus employ a new special action $\bullet$. The left-hand side then first requires a $\bullet$-transition into $n$ different states $s_{i}$, each requiring the above formula for its respective $i$.

Proposition 4.15 Modal refinement is $\Pi_{2}^{\mathrm{P}}$-hard even if left-hand side is in positive $D N F$ and right-hand side is in positive $C N F$.

Proof The proof is done by reduction from the validity of the quantified Boolean formula $\forall x_{1}, \ldots, x_{n} \exists y_{1}, \ldots, y_{m}: \varphi\left(x_{1}, \ldots, x_{n}, y_{1}, \ldots, y_{m}\right)$, where $\varphi$ is in CNF.

Let the action alphabet be $\Sigma=\left\{t_{1}, \ldots, t_{n}, f_{1}, \ldots, f_{n}, \bullet\right\}$. The two systems $(S, T, P, \Phi)$ and $\left(S^{\prime}, T^{\prime}, P^{\prime}, \Phi^{\prime}\right)$ are built as follows: $S=\left\{s, s^{\prime}\right\} \cup\left\{s_{i} \mid 1 \leq i \leq n\right\}$, $T=\left\{\left(s, \bullet, s_{i}\right),\left(s_{i}, t_{i}, s^{\prime}\right),\left(s_{i}, f_{i}, s^{\prime}\right) \mid 1 \leq i \leq n\right\}, P=\left\{x_{1}, \ldots, x_{n}\right\}, \Phi(s)=\bigwedge_{i}\left(\bullet, s_{i}\right)$, $\Phi\left(s_{i}\right)=\left(x_{i} \wedge\left(t_{i}, s^{\prime}\right)\right) \vee\left(\neg x_{i} \wedge\left(f_{i}, s^{\prime}\right)\right), \Phi\left(s^{\prime}\right)=\mathbf{t t} ; S^{\prime}=\left\{t, t^{\prime}\right\} \cup\left\{u_{i}, v_{i} \mid 1 \leq i \leq\right.$ $n\}, T^{\prime}=\left\{\left(t, \bullet, u_{i}\right),\left(t, \bullet, v_{i}\right),\left(u_{i}, t_{i}, t^{\prime}\right),\left(u_{i}, f_{i}, t^{\prime}\right),\left(v_{i}, f_{i}, t^{\prime}\right),\left(v_{i}, t_{i}, t^{\prime}\right) \mid 1 \leq i \leq n\right\}$, $P^{\prime}=\left\{y_{1}, \ldots, y_{n}\right\}, \Phi^{\prime}(t)=\varphi\left[\left(\bullet, u_{i}\right) / x_{i},\left(\bullet, v_{i}\right) / \neg x_{i}\right], \Phi^{\prime}\left(u_{i}\right)=\left(t_{i}, t^{\prime}\right), \Phi^{\prime}\left(v_{i}\right)=\left(f_{i}, t^{\prime}\right)$, $\Phi^{\prime}\left(t^{\prime}\right)=\mathbf{t t}$.

Now $\forall x \exists y: \varphi(x, y)$ holds if and only if $s \leq_{\mathrm{m}} t$. The reasoning behind this fact is similar to the proof of Proposition 4.13

The proof of the next proposition is a slight alteration of previous proof where the $\bullet$-construction is performed in two steps. 
Proposition 4.16 Modal refinement is $\Pi_{2}^{\mathrm{P}}$-hard even if left-hand side is in positive $D N F$ and right-hand side is in positive DNF.

Proof The proof is done by reduction from the validity of the quantified Boolean formula $\forall x_{1}, \ldots, x_{n} \exists y_{1}, \ldots, y_{m}: \varphi\left(x_{1}, \ldots, x_{n}, y_{1}, \ldots, y_{m}\right)$, where $\varphi$ is in CNF. Let $\varphi_{1}, \ldots, \varphi_{k}$ denote the clauses of $\varphi$.

Let the action alphabet be $\Sigma=\left\{t_{1}, \ldots, t_{n}, f_{1}, \ldots, f_{n}, \bullet\right\}$. The two systems $(S, T, P, \Phi)$ and $\left(S^{\prime}, T^{\prime}, P^{\prime}, \Phi^{\prime}\right)$ are built as follows: $S=\left\{s, s^{\prime}, s^{\prime \prime}\right\} \cup\left\{s_{i} \mid 1 \leq\right.$ $i \leq n\}, T=\left\{\left(s, \bullet, s^{\prime}\right)\right\} \cup\left\{\left(s^{\prime}, \bullet, s_{i}\right),\left(s_{i}, t_{i}, s^{\prime \prime}\right),\left(s_{i}, f_{i}, s^{\prime \prime}\right) \mid 1 \leq i \leq n\right\}, P=$ $\left\{x_{1}, \ldots, x_{n}\right\}, \Phi(s)=\left(\bullet, s^{\prime}\right), \Phi\left(s^{\prime}\right)=\bigwedge_{i}\left(\bullet, s_{i}\right), \Phi\left(s_{i}\right)=\left(x_{i} \wedge\left(t_{i}, s^{\prime \prime}\right)\right) \vee\left(\neg x_{i} \wedge\right.$ $\left.\left(f_{i}, s^{\prime \prime}\right)\right), \Phi\left(s^{\prime \prime}\right)=\mathbf{t t} ; S^{\prime}=\left\{t, t^{\prime}\right\} \cup\left\{u_{i}, v_{i} \mid 1 \leq i \leq n\right\} \cup\left\{w_{j} \mid 1 \leq j \leq k\right\}$, $T^{\prime}=\left\{\left(t, \bullet, w_{j}\right) \mid 1 \leq j \leq k\right\} \cup\left\{\left(w_{j}, \bullet, u_{i}\right),\left(w_{j}, \bullet, v_{i}\right) \mid 1 \leq i \leq n, 1 \leq j \leq\right.$ $k\} \cup\left\{\left(u_{i}, t_{i}, t^{\prime}\right),\left(u_{i}, f_{i}, t^{\prime}\right),\left(v_{i}, f_{i}, t^{\prime}\right),\left(v_{i}, t_{i}, t^{\prime}\right) \mid 1 \leq i \leq n\right\}, P^{\prime}=\left\{y_{1}, \ldots, y_{n}\right\}$, $\Phi^{\prime}(t)=\bigwedge_{j} w_{j}, \Phi^{\prime}\left(w_{j}\right)=\varphi_{j}^{\prime}$ where $\varphi_{j}^{\prime}$ is created from $\varphi_{j}$ by changing all positive literals $x_{i}$ into $\left(\bullet, u_{i}\right)$ and all negative literals $\neg x_{i}$ into $\left(\bullet, v_{i}\right) . \Phi^{\prime}\left(u_{i}\right)=\left(t_{i}, t^{\prime}\right)$, $\Phi^{\prime}\left(v_{i}\right)=\left(f_{i}, t^{\prime}\right), \Phi^{\prime}\left(t^{\prime}\right)=\mathbf{t t}$.

Now $\forall x \exists y: \varphi(x, y)$ holds if and only if $s \leq_{\mathrm{m}} t$.

The proof of the third proposition is a combination of DNF-binding (including the $\bullet$-construction) with the previously used $*$-construction.

Proposition 4.17 Modal refinement is $\Pi_{4}^{\mathrm{P}}$-hard even if the left-hand side is in positive $D N F$.

Proof The proof is done by reduction from the validity of the quantified Boolean formula $\forall x \exists y \forall z \exists w: \varphi(x, y, z, w)$ where $x, y, z, w$ are all $n$-dimensional binary vectors and $\varphi$ is in CNF.

We let $\Sigma=\left\{t_{1}, \ldots, t_{n}, f_{1}, \ldots, f_{n}, z_{1}, \ldots, z_{n}, *, \bullet\right\}$ and we create the two systems $(S, T, P, \Phi),\left(S^{\prime}, T^{\prime}, P^{\prime}, \Phi^{\prime}\right)$ over the action alphabet $\Sigma$ as follows:

$S=\left\{s, s^{\prime}\right\} \cup\left\{s_{i} \mid 1 \leq i \leq n\right\}, T=\left\{\left(s, \bullet, s_{i}\right),\left(s_{i}, t_{i}, s^{\prime}\right),\left(s_{i}, f_{i}, s^{\prime}\right),\left(s, z_{i}, s^{\prime}\right) \mid\right.$ $1 \leq i \leq n\} \cup\left\{\left(s, *, s^{\prime}\right)\right\}, P=\left\{x_{1}, \ldots, x_{n}\right\}, \Phi(s)=\left(*, s^{\prime}\right) \wedge \bigwedge_{i}\left(\bullet, s_{i}\right), \Phi\left(s_{i}\right)=$ $\left(x_{i} \wedge\left(t_{i}, s^{\prime}\right)\right) \vee\left(\neg x_{i} \wedge\left(f_{i}, s^{\prime}\right)\right)$ for all $1 \leq i \leq n, \Phi\left(s^{\prime}\right)=\mathbf{t t}$;

$S^{\prime}=\left\{t, t^{\prime}\right\} \cup\left\{u_{i}, v_{i}, w_{i} \mid 1 \leq i \leq n\right\}, T^{\prime}=\left\{\left(t, z_{i}, t^{\prime}\right),\left(t, \bullet, u_{i}\right),\left(t, \bullet, v_{i}\right),\left(t, *, w_{i}\right)\right.$, $\left.\left(u_{i}, t_{i}, t^{\prime}\right),\left(u_{i}, f_{i}, t^{\prime}\right),\left(v_{i}, t_{i}, t^{\prime}\right),\left(v_{i}, f_{i}, t^{\prime}\right) \mid 1 \leq i \leq n\right\} \cup\left\{\left(t, *, t^{\prime}\right)\right\}, P^{\prime}=\left\{y_{1}, \ldots, y_{n}\right\}$, $\Phi^{\prime}(t)=\left(*, t^{\prime}\right) \wedge \varphi\left[\left(\bullet, u_{i}\right) / x_{i},\left(\bullet, v_{i}\right) / \neg x_{i},\left(z_{i}, t^{\prime}\right) / z_{i},\left(*, w_{i}\right) / w_{i}\right]$, for all $1 \leq i \leq n$ : $\Phi^{\prime}\left(u_{i}\right)=\left(t_{i}, t^{\prime}\right), \Phi^{\prime}\left(v_{i}\right)=\left(f_{i}, t^{\prime}\right), \Phi^{\prime}\left(w_{i}\right)=\Phi^{\prime}\left(t^{\prime}\right)=\mathbf{t t}$.

It can be verified, using similar arguments as before, that $s \leq_{\mathrm{m}} t$ if and only if $\forall x \exists y \forall z \exists w: \varphi(x, y, z, w)$.

The following proposition solves the only remaining case.

Proposition 4.18 Modal refinement is $\Pi_{3}^{\mathrm{P}}$-hard even if the right-hand side is in positive CNF.

Proof We prove the hardness by reduction from the validity of the $\mathrm{QBF}_{3}^{\forall}$ formula $\forall x \exists y \forall z: \varphi(x, y, z)$ where $x, y, z$ are vectors of size $n$. We construct two PMTSs $(S, T, P, \Phi)$ and $\left(S^{\prime}, T^{\prime}, P^{\prime}, \Phi^{\prime}\right)$ as follows:

1. $P=\left\{x_{1}, \ldots, x_{n}\right\}, S=\left\{s, s^{\prime}, s_{1}, \ldots, s_{n}\right\}$,

$T=\left\{\left(s, t_{i}, s^{\prime}\right),\left(s, f_{i}, s^{\prime}\right),\left(s, *, s_{i}\right) \mid 1 \leq i \leq n\right\} \cup\left\{\left(s, *, s^{\prime}\right)\right\}$,

$\Phi(s)=\neg \varphi\left[\left(t_{i}, s^{\prime}\right) / y_{i},\left(*, s_{i}\right) / z_{i}\right] \wedge \bigwedge_{i}\left(\left(t_{i}, s^{\prime}\right) \Leftrightarrow \neg\left(f_{i}, s^{\prime}\right)\right) \wedge\left(*, s^{\prime}\right)$,

$\Phi\left(s^{\prime}\right)=\Phi\left(s_{i}\right)=\mathbf{t t}$ for all $i$. 
2. $P^{\prime}=\left\{y_{1}, \ldots, y_{n}\right\}, S^{\prime}=\left\{t, t^{\prime}, u_{1}, \ldots, u_{n}, v_{1}, \ldots, v_{n}\right\}$,

$T^{\prime}=\left\{\left(t, *, t^{\prime}\right)\right\} \cup\left\{\left(t, t_{i}, t^{\prime}\right),\left(t, f_{i}, t^{\prime}\right),\left(t, t_{i}, u_{i}\right),\left(t, f_{i}, v_{i}\right),\left(u_{i}, b, t^{\prime}\right),\left(v_{i}, b, t^{\prime}\right) \mid 1 \leq\right.$ $i \leq n\}, \Phi^{\prime}(t)=\bigvee_{i}\left(\left(t_{i}, u_{i}\right) \vee\left(f_{i}, v_{i}\right)\right), \Phi^{\prime}\left(u_{i}\right)=y_{i} \Rightarrow\left(b, t^{\prime}\right)$ for all $i, \Phi^{\prime}\left(v_{i}\right)=$ $\neg y_{i} \Rightarrow\left(b, t^{\prime}\right)$ for all $i, \Phi^{\prime}\left(t^{\prime}\right)=\mathbf{t t}$.

(Clearly, this is in positive CNF. In fact, we only use disjunctions here.)

To make our first observation, we use the following notation: By $s \leq_{\mathrm{m}}^{\mu, \nu} t$ we denote the fact that there exists a modal refinement respecting $\mu$ and $\nu$ containing $(s, t)$. We now observe that for every choice of $\mu \subseteq P$ and $\nu \subseteq P^{\prime}, s^{\prime} \leq \stackrel{\mu, \nu}{\mathrm{m}} u_{i}$ iff $y_{i} \notin \nu$. Similarly, $s^{\prime} \leq{ }_{\mathrm{m}}^{\mu, \nu} v_{i}$ iff $y_{i} \in \nu$.

Assume now that $\forall x \exists y \forall z: \varphi(x, y, z)$ is true. We want to show that $s \leq_{\mathrm{m}} t$. Let $\mu \subseteq P$ be arbitrary. Let us fix the valuation $\hat{x}$ of $x$ given by $\mu$. We know that $\exists y \forall z: \varphi(\hat{x}, y, z)$ holds. Let us fix such valuation of $y$ and call it $\hat{y}$. Let us further choose $\nu=\left\{y_{i} \mid \hat{y}_{i}=\mathbf{t t}\right\}$. Let now $M \in \operatorname{Tran}_{\mu}(s)$ be arbitrary. Clearly, $\left(t_{i}, s^{\prime}\right) \in M$ iff $\left(f_{i}, s^{\prime}\right) \notin M$. Furthermore $M$ induces a valuation $\tilde{y}$ of $y$ (via the choice of $t_{i} / f_{i}$ ) and $\tilde{z}$ of $z$ (via the choice of $\left(*, s_{i}\right)$ ), such that $\neg \varphi(\hat{x}, \tilde{y}, \tilde{z})$ holds.

As we know that $\forall z: \varphi(\hat{x}, \hat{y}, z)$ holds, this means that $\hat{y}$ and $\tilde{y}$ differ. Therefore, there is at least one index $j$ such that $\hat{y}_{j} \neq \tilde{y}_{j}$ and thus either $y_{j} \in \nu$ and $\left(f_{j}, s^{\prime}\right) \in M$ or $y_{j} \notin \nu$ and $\left(t_{j}, s^{\prime}\right) \in M$.

To show that $s \leq_{\mathrm{m}} t$, we use Lemma 2.5. Clearly, Condition (1) holds. We now show that $\operatorname{match}_{t}(M) \in \operatorname{Tran}_{\nu}(t)$. Clearly, $\operatorname{match}_{t}(M)=\left\{\left(*, t^{\prime}\right)\right\} \cup\left\{\left(t_{i}, t^{\prime}\right) \mid\left(t_{i}, s^{\prime}\right) \in\right.$ $M\} \cup\left\{\left(f_{i}, t^{\prime}\right) \mid\left(f_{i}, s^{\prime}\right) \in M\right\} \cup\left\{\left(t_{i}, u_{i}\right) \mid\left(t_{i}, s^{\prime}\right) \in M\right.$ and $\left.y_{i} \notin \nu\right\} \cup\left\{\left(f_{i}, v_{i}\right) \mid\left(f_{i}, s^{\prime}\right) \in M\right.$ and $\left.y_{i} \in \nu\right\}$. Due to our observation that $\tilde{y}$ and $\hat{y}$ differ, at least one of $\left(t_{i}, u_{i}\right)$ or $\left(f_{i}, v_{i}\right)$ is contained in $\operatorname{match}_{t}(M)$. Therefore, $\operatorname{match}_{t}(M) \in \operatorname{Tran}_{\nu}(t)$ and thus $s \leq \mathrm{m} t$

Let us now assume that $\forall x \exists y \forall z: \varphi(x, y, z)$ does not hold. We show that $s \not \underline{\mathrm{m}}_{\mathrm{m}} t$. Our assumption means that $\exists x \forall y \exists z: \neg \varphi(x, y, z)$ holds. Let us fix such a valuation $\hat{x}$ of $x$ and choose $\mu=\left\{x_{i} \mid \hat{x}_{i}=\mathbf{t t}\right\}$. Let $\nu \subseteq P^{\prime}$ be arbitrary and let us fix the valuation $\hat{y}$ of $y$ represented by $\nu$. Let us further fix a valuation $\hat{z}$ of $z$ such that $\neg \varphi(\hat{x}, \hat{y}, \hat{z})=\mathbf{t t}$. Choose $M=\left\{\left(t_{i}, s^{\prime}\right) \mid \hat{y}_{i}=\mathbf{t t}\right\} \cup\left\{\left(f_{i}, s^{\prime}\right) \mid \hat{y}_{i} \neq \mathbf{t t}\right\} \cup\left\{\left(*, s_{i}\right) \mid \hat{z}_{i}=\right.$ tt $\} \cup\left\{\left(*, s^{\prime}\right)\right\}$. Clearly, $M \in \operatorname{Tran}_{\mu}(s)$.

We now show that $\operatorname{match}_{t}(M) \notin \operatorname{Tran}_{\nu}(t)$. To be in $\operatorname{Tran}_{\nu}(t)$ a set has to contain at least one of $\left(t_{i}, u_{i}\right)$ or $\left(f_{i}, v_{i}\right)$. However, $\operatorname{match}_{t}(M)$ does not contain any of these, as $M$ contains $\left(t_{i}, s^{\prime}\right)$ iff $y_{i} \in \nu$ and $\left(f_{i}, s^{\prime}\right)$ iff $y_{i} \notin \nu$. (See our observation about $s^{\prime} \leq{ }_{\mathrm{m}}^{\mu, \nu} u_{i}$ and $s^{\prime} \leq_{\mathrm{m}}^{\mu, \nu} v_{i}$ above.) Therefore, $s \underline{x}_{\mathrm{m}} t$.

Although the complexity may seem discouraging in many cases, there is an important remark to make. The refinement checking may be exponential, but only in the outdegree of each state and the number of parameters, while it is polynomial in the number of states. As one may expect the outdegree and the number of parameters to be much smaller than the number of states, this means that the refinement checking may still be done in a rather efficient way. This claim is furthermore supported by the existence of efficient SAT solvers that may be employed to check the inner conditions in the modal refinement.

\section{Modal Refinement Checking by Reduction to QBF}

In this section, we show how to solve the modal refinement problem on parameterfree PMTS and on general PMTS using QBF solvers. Although modal refinement 
is computationally hard $\left(\Pi_{2}^{\mathrm{P}}\right.$-complete on parameter-free PMTS and $\Pi_{4}^{\mathrm{P}}$-complete on PMTS), by using QBF solvers we obtain a feasible method for modal refinement checking, as documented by our preliminary experiments.

As mentioned, in order to decide whether modal refinement holds between two states, a reduction to a quantified Boolean formula will be used. Recall the definition of the problem $\mathrm{QBF}_{n}^{Q}$ where $Q \in\{\exists, \forall\}$ given in Definition 4.1 .

\subsection{Construction for parameter-free PMTS}

Due to the completeness of QBF problems and our result in Proposition 4.4 it is possible to polynomially reduce modal refinement on parameter-free PMTS to $\mathrm{QBF}_{2}^{\forall}$. However, following the construction in Proposition 4.4 we have to perform fixed-point iterations in order to compute the refinement relation, resulting in numerous invocations of the external QBF solver. Additionally, this approach is not applicable in the PMTS case. We shall instead provide a direct reduction of modal refinement checking to $\mathrm{QBF}_{3}^{\exists}$.

Let $s \in S_{1}$ and $t \in S_{2}$ be states of two arbitrary parameter-free PMTSs $\mathcal{M}_{1}=\left(S_{1}, T_{1}, \emptyset, \Phi_{1}\right)$ and $\mathcal{M}_{2}=\left(S_{2}, T_{2}, \emptyset, \Phi_{2}\right)$. Furthermore let

$$
A p=\underbrace{\left(S_{1} \times S_{2}\right)}_{X_{R}} \uplus \underbrace{T_{1}}_{X_{T_{1}}} \uplus \underbrace{\left(S_{1} \times T_{2}\right)}_{X_{T_{2}}}
$$

be a set of atomic propositions. The intended meaning is that $(u, v) \in X_{R}$ is assigned tt if and only if it is also contained in the modal refinement relation $R$, while $X_{T_{1}}$ and $X_{T_{2}}$ encode transitions. A state from $S_{1}$ is attached to the set $T_{2}$, because the variables used to encode $N \in \operatorname{Tran}(t)$ with $t \in S_{2}$ must be unique for different states of $S_{1}$ in order to move the $\exists$ quantification over conjuncts in the formula.

We now construct a formula $\Psi_{s, t} \in \mathcal{B}(A p)$ satisfying

$$
s \leq_{m} t \quad \text { iff } \quad \exists X_{R} \forall X_{T_{1}} \exists X_{T_{2}} \Psi_{s, t} \in \mathrm{QBF}_{3}^{\exists} .
$$

To this end, we shall use a macro $\psi_{u, v}$ capturing the condition which has to be satisfied by any element $(u, v) \in R$. Furthermore, we ensure that $(s, t)$ is assigned tt by every satisfying assignment for the formula by placing it directly in the conjunction:

$$
\Psi_{s, t}=(s, t) \wedge \bigwedge_{(u, v) \in X_{R}}\left((u, v) \Rightarrow \psi_{u, v}\right) .
$$

It remains to define the formula $\psi_{u, v}$ that should guarantee that $(u, v) \in R$. We start with the modal refinement condition as a blueprint:

$$
\begin{aligned}
\forall M \in \operatorname{Tran}(u): \exists N \in \operatorname{Tran}(v): & \forall\left(a, u^{\prime}\right) \in M: \exists\left(a, v^{\prime}\right) \in N:\left(u^{\prime}, v^{\prime}\right) \in R \wedge \\
& \forall\left(a, v^{\prime}\right) \in N: \exists\left(a, u^{\prime}\right) \in M:\left(u^{\prime}, v^{\prime}\right) \in R .
\end{aligned}
$$

As $M$ and $N$ are subsets of $T_{1}(u)$ and $T_{2}(v)$, respectively, and are finite, the inner quantifiers can be expanded causing only a polynomial growth of the formula size. Further, Tran sets are replaced by the original definition and the outer quantifiers 
are moved in front of $\Psi_{s, t}$. As the state obligations are defined over a different set of atomic propositions $(\Phi(v) \in \mathcal{B}((\Sigma \times S) \cup P) \not \subset \mathcal{B}(A p))$, a family of mapping functions $\pi_{x}$ is introduced where $x$ is either a state from $S_{1}$ or a pair of states from $S_{1} \times S_{2}$.

$$
\begin{aligned}
\pi_{x}: \mathcal{B}(\Sigma \times S) & \rightarrow \mathcal{B}(A p) \\
\mathbf{t t} & \mapsto \mathbf{t t} \\
(a, v) & \mapsto(x, a, v) \quad \text { for } a \in \Sigma, v \in S \\
\neg \varphi & \mapsto \neg \pi_{x}(\varphi) \\
\varphi_{1} \wedge \varphi_{2} & \mapsto \pi_{x}\left(\varphi_{1}\right) \wedge \pi_{x}\left(\varphi_{2}\right) \\
\varphi_{1} \vee \varphi_{2} & \mapsto \pi_{x}\left(\varphi_{1}\right) \vee \pi_{x}\left(\varphi_{2}\right)
\end{aligned}
$$

Applying these steps to the blueprint yields the following result:

$$
\begin{aligned}
& \psi_{u, v}= \pi_{u}\left(\Phi_{1}(u)\right) \Rightarrow \pi_{u, v}\left(\Phi_{2}(v)\right) \wedge \varphi_{u, v} \\
& \varphi_{u, v}\left.=\bigwedge_{\begin{array}{c}
u^{*} \in X_{T_{1}} \\
u^{*}=\left(u, a, u^{\prime}\right) v^{*}=\left(u, v, a, v^{\prime}\right) \\
v^{*} \in X_{T_{2}}
\end{array}}\left(v^{*} \wedge\left(u^{\prime}, v^{\prime}\right)\right)\right) \\
& \wedge \bigwedge\left(v^{*} \Rightarrow \bigvee_{v^{*} \in X_{T_{2}}, u^{*} \in X_{T_{1}}}\left(u^{*} \wedge\left(u^{\prime}, v^{\prime}\right)\right)\right) . \\
& v^{*}=\left(u, v, a, v^{\prime}\right) u^{*}=\left(u, a, u^{\prime}\right)
\end{aligned}
$$

Theorem 5.1 For states $s, t$ of a parameter-free PMTS, we have

$$
s \leq_{m} t \quad \text { iff } \quad \exists X_{R} \forall X_{T_{1}} \exists X_{T_{2}} \Psi_{s, t} \in \mathrm{QBF}_{3}^{\exists} .
$$

Before proving the soundness and the correctness of the construction for parameter-free PMTS, a lemma is introduced to simplify this proof.

Lemma 5.2 Let $(u, v) \in S_{1} \times S_{2}$ be a pair of states. Let $\mathcal{A}_{X_{R}}, \mathcal{A}_{X_{T_{1}}}$ and $\mathcal{A}_{X_{T_{2}}}$ be partial valuations for the respective sets of atomic propositions that appear in the indices. Furthermore let $R \subseteq S_{1} \times S_{2}, M \in \operatorname{Tran}_{\emptyset}(u)$ and $N \in \operatorname{Tran}_{\emptyset}(v)$ such that $\mathcal{A}_{X_{R}}=R, \mathcal{A}_{X_{T_{1}}} \supseteq \pi_{u}(M)$ and $\mathcal{A}_{X_{T_{2}}} \supseteq \pi_{u, v}(N)$. Then $\mathcal{A}_{X_{R}} \cup \mathcal{A}_{X_{T_{1}}} \cup \mathcal{A}_{X_{T_{2}}} \models \varphi_{u, v}$ if and only if

$$
\begin{aligned}
R \cup M \cup N \models & \forall\left(a, s^{\prime}\right) \in M: \exists\left(a, t^{\prime}\right) \in N:\left(s^{\prime}, t^{\prime}\right) \in R \\
& \wedge \forall\left(a, t^{\prime}\right) \in N: \exists\left(a, s^{\prime}\right) \in M:\left(s^{\prime}, t^{\prime}\right) \in R .
\end{aligned}
$$

Proof We assume the conditions of the lemma, the set $\mathcal{A}_{X}=\mathcal{A}_{X_{R}} \cup \mathcal{A}_{X_{T_{1}}} \cup \mathcal{A}_{X_{T_{2}}}$ and $\mathcal{A}_{R}=R \cup M \cup N$. Additionally, we only consider one half of the conjunction, as the other one is proven analogously.

$$
\begin{aligned}
& \mathcal{A}_{R} \models \\
& \mathcal{A}_{R}=\bigwedge_{\left(a, s^{\prime}\right) \in T_{1}(s)}\left(\left(a, s^{\prime}\right) \in M \Rightarrow \bigvee_{\left(a, t^{\prime}\right) \in T_{2}(t)}\left(\left(a, t^{\prime}\right) \in N \wedge\left(\left(s^{\prime}, t^{\prime}\right) \in R\right)\right)\right) \\
& \text { iff } \quad \mathcal{A}_{X}=\bigwedge_{\substack{s^{*} \in X_{T_{1}} \\
s^{*}=\left(s, a, s^{\prime}\right) t^{*}=\left(s, t, a, t^{\prime}\right)}}\left(s^{*} \Rightarrow \bigvee_{X^{*}}\left(t^{*} \wedge\left(s^{\prime}, t^{\prime}\right)\right)\right)
\end{aligned}
$$


As $M$ and $N$ are finite sets, $\forall$ and $\exists$ quantifiers may simply be expanded. In the second step, we apply $\pi_{x}$ and substitute $\in$ with atomic propositions.

Soundness and Completeness of Theorem 5.1 For soundness, assume $s \leq_{m} t$ with the modal refinement relation $R$. As the partial valuation for $X_{R}$, we set $\mathcal{A}_{X_{R}}=R$. Furthermore let $\mathcal{A}_{X_{T_{1}}} \subseteq X_{T_{1}}$ be an arbitrary assignment. We now construct an assignment $\mathcal{A}_{X_{T_{2}}}$, such that

$$
\mathcal{A}=\mathcal{A}_{X_{R}} \cup \mathcal{A}_{X_{T_{1}}} \cup \mathcal{A}_{X_{T_{2}}}=\Psi_{s, t}
$$

holds. Without adding anything to $\mathcal{A}_{X_{T_{2}}}$, clearly $\mathcal{A}=(s, t)$ and $\mathcal{A}=(u, v) \Rightarrow \psi_{u, v}$ for all $(u, v) \in X_{R} \cap \bar{R}$ hold.

Let now $(u, v) \in R$ be an arbitrary pair of states. If $\mathcal{A} \not \models \pi_{u}(\Phi(u))$, then $\mathcal{A}=\psi_{u, v}$ and $\mathcal{A}=(u, v) \Rightarrow \psi_{u, v}$. Hence we assume now $\mathcal{A} \models \pi_{u}(\Phi(u))$. Since $(u, v) \in R$, there exists for all $M \in \operatorname{Tran}_{\emptyset}(u)$ a set $N$, such that the condition holds, which is included in the assignment $\mathcal{A}_{X_{T_{2}}} \supseteq \pi_{u, v}(N)$. This can safely be done due to the prefixing and with Lemma 5.2 we get $\mathcal{A}=\varphi_{u, v}$ and $\mathcal{A}=(u, v) \Rightarrow \psi_{u, v}$.

As a valuation $\mathcal{A}$ can be constructed for a fixed modal refinement relation, such that for all subsets of $X_{T_{1}}$ it satisfies the formula, $\exists X_{R} \forall X_{T_{1}} \exists X_{T_{2}} \Psi_{s, t} \in \mathrm{QBF}_{3}^{\exists}$ holds.

For completeness, we now assume

$$
\exists X_{R} \forall X_{T_{1}} \exists X_{T_{2}} \Psi_{s, t} \in \mathrm{QBF}_{3}^{\exists} .
$$

Then there exists a partial valuation $\mathcal{A}_{X_{R}} \subseteq \mathcal{A}$ for $X_{R}$, which satisfies $\Psi_{s, t} . R$ is simply constructed by setting $R=\mathcal{A}_{X_{R}}$. Clearly $(s, t) \in R$. Let now $(u, v) \in R$ be an arbitrary pair of states. As (5.4) is satisfied for this pair, either $\Phi(u)$ is unsatisfiable and there simply exists no $M \in \operatorname{Tran}_{\emptyset}(s)$ or for the chosen $M=\pi_{u}^{-1}\left(\mathcal{A}_{X_{T_{1}}}\right)$ exists a $N=\pi_{u, v}^{-1}\left(\mathcal{A}_{X_{T_{2}}}\right)$. By Lemma 5.2 the modal refinement condition holds for this arbitrary pair. Hence $R$ is a modal refinement relation.

Finally, we show that the reduction indeed takes only polynomial time. For this observe that (5.5) is in $\mathcal{O}\left(\left|T_{1}(u)\right|\left|T_{2}(v)\right|\right)$. Therefore (5.4) is in $\mathcal{O}\left(\left|T_{1}(u)\right|\left|T_{2}(v)\right|+\right.$ $\left.\left|\Phi_{1}(u)\right|+\left|\Phi_{2}(v)\right|\right)$. Leading to a total formula size of

$$
\mathcal{O}\left(\left|S_{1}\right|\left|S_{2}\right|\left(\left|T_{1}\right|\left|T_{2}\right|+\left|\Phi_{1}\right|+\left|\Phi_{2}\right|\right)\right)
$$

\subsection{Construction for PMTS}

We now reduce the modal refinement on PMTS to $\mathrm{QBF}_{4}^{\forall}$, which corresponds directly to the complexity result for this problem proved in the present article. Nevertheless, due to the first existential quantification in $\forall \exists \forall \exists$ alternation sequence, we can still guess the refinement relation using the QBF solver rather than to do the lengthy fixed-point computation.

In the PMTS case, we have to find for all parameter valuations for the system of $s$ a valuation for the system of $t$, such that there exists a modal refinement relation containing $(s, t)$. We simply choose universally a valuation for the parameters of the left system (the underlying system of $s$ ) and then existentially for the right system (the underlying system of $t$ ). Prior to checking modal refinement, the valuations 
are fixed, so the PMTS becomes a parameter-free PMTS. This is accomplished by extending $A p$ with $P_{1}$ and $P_{2}$ and adding the necessary quantifiers to the formula. Thus we obtain the following:

Theorem 5.3 For states $s, t$ of a PMTS, we have

$$
s \leq_{m} t \quad \text { iff } \quad \forall P_{1} \exists P_{2} \exists X_{R} \forall X_{T_{1}} \exists X_{T_{2}} \Psi_{s, t} \in \mathrm{QBF}_{4}^{\forall} .
$$

\subsection{Experimental Results}

We now show how our method performs in practice by implementing the reduction and linking it to the QBF solver Quantor ${ }^{2}$. In order to evaluate whether our solution scales, we generated several random samples of MTS and parametric MTS with a different number of parameters ranging from 0 to 20 (as displayed in Table 5.1 in parentheses). The systems use two different actions and the edges were generated randomly so that they create a tree with some additional "noise" edges to reach the specified number of outgoing transitions. In order to simulate a step-wise refinement, we took these randomly generated systems and performed a few randomised operations, such as insertion and removal of actions, states and transitions, as well as changes to the transition labels or to the obligation function of a state. Table 5.1 is split according to the instances where the modal refinement (after a few randomised changes were performed) remained satisfied and where it became unsatisfied. The degree of the random graph is the number of outgoing transitions from each state. Given the fixed two-letter action alphabet, the higher the degree the more nondeterminism is present in the generated systems.

The entries in the tables are average running times in seconds with around $90 \%$ of the time used by the external QBF solver. The standard deviation in our experiments ranged from 10 to $60 \%$, a few exceptions are noted with a footnote. The reason why the refinement checking takes occasionally significantly more time is likely due to the fact that the generated graphs are hard instances for the QBF solver (for the chosen heuristic). The experiments were performed on an Intel Core i7 (2.7 Ghz) with 16 GB RAM using Java 1.8.

On the one hand, we can observe that the number of parameters does not generally play any major role in the running time, excluding some rare cases. The running times on PMTS with 20 parameters are close to parameter-free PMTS. Therefore, the greatest theoretical complexity threat - the number of parameters requiring in general a search through exponentially many combinations - seems to be in practice eliminated by the use of QBF solvers.

On the other hand, observe that the running time is affected by the level of nondeterminism (degree). This effect is more emphasised as the number of parameters increases. However, the level of nondeterminism in many applications is often quite low [18, hence this dependency does not pose a serious problem in practice.

The method is implemented within the tool MoTraS [37], which is equipped with a graphical as well as a command-line interface. More information on the tool and the data used to perform this experimental evaluation can be found at https://www7.in.tum.de/ kretinsk/motras.html.

\footnotetext{
2 http://fmv.jku.at/quantor/
} 
Table 5.1: Experimental results: degree is the number of outgoing transitions and the size of the problem is the number of states

\begin{tabular}{lrrrrrr} 
degree 2 (satisfied) & 50 & 100 & 150 & 200 & 250 & 300 \\
\hline MTS & 0.17 & 0.36 & 0.82 & 1.44 & 2.31 & 3.4 \\
PMTS (0) & 0.08 & 0.34 & 0.79 & 1.45 & 2.42 & 3.23 \\
PMTS (1) & 0.08 & 0.39 & 0.93 & 1.69 & 2.70 & 3.82 \\
PMTS (5) & 0.09 & 0.42 & 0.92 & 1.79 & 2.79 & 3.91 \\
PMTS (20) & 0.09 & 0.57 & 1.11 & 4.53 & $32.38 \sqrt{3}$ & $37.85{ }^{4}$ \\
degree 2 (unsatisfied) & 50 & 100 & 150 & 200 & 250 & 300 \\
\hline MTS & 0.09 & 0.36 & 0.81 & 1.50 & 2.34 & 3.21 \\
PMTS (0) & 0.08 & 0.36 & 0.78 & 1.51 & 2.38 & 3.43 \\
PMTS (1) & 0.09 & 0.39 & 0.82 & 1.62 & 2.71 & 3.74 \\
PMTS (5) & 0.09 & 0.39 & 0.89 & 1.66 & 2.83 & 3.80 \\
PMTS (20) & 0.09 & 0.42 & 0.97 & 2.01 & 2.93 & 4.32
\end{tabular}

\begin{tabular}{lrrrrrr} 
degree 3 (satisfied) & 50 & 100 & 150 & 200 & 250 & 300 \\
\hline MTS & 0.31 & 0.94 & 2.22 & 4.03 & 6.59 & 9.48 \\
PMTS (0) & 0.20 & 0.91 & 2.23 & 4.00 & 6.65 & 9.56 \\
PMTS (1) & 0.27 & 1.04 & 2.73 & 4.60 & 7.79 & 11.61 \\
PMTS (5) & 0.24 & 1.10 & 2.73 & 5.05 & 8.21 & 12.19 \\
PMTS (20) & 0.34 & 1.34 & 3.06 & 6.34 & 11.21 & $227.55[5$ \\
degree 3 (unsatisfied) & 50 & 100 & 150 & 200 & 250 & 300 \\
\hline MTS & 0.20 & 0.82 & 2.13 & 3.79 & 6.36 & 8.85 \\
PMTS (0) & 0.21 & 0.87 & 2.17 & 4.05 & 6.59 & 8.85 \\
PMTS (1) & 0.26 & 0.99 & 2.48 & 4.55 & 7.46 & 10.99 \\
PMTS (5) & 0.28 & 1.13 & 2.83 & 4.96 & 8.42 & 11.63 \\
PMTS (20) & 0.28 & 1.11 & 3.22 & 5.83 & 8.98 & 12.69
\end{tabular}

\begin{tabular}{lrrrrrr} 
degree 5 (satisfied) & 50 & 100 & 150 & 200 & 250 & 300 \\
\hline MTS & 0.79 & 2.89 & 6.69 & 12.88 & 19.79 & 30.27 \\
PMTS (0) & 0.89 & 3.54 & 8.64 & 16.83 & 26.29 & 38.27 \\
PMTS (1) & 2.24 & 8.86 & 22.02 & 41.94 & 81.86 & 113.59 \\
PMTS (5) & 3.32 & 8.64 & 16.91 & 38.17 & 58.31 & 95.79 \\
PMTS (20) & 1.42 & 7.59 & 21.69 & 80.296 & 61.84 & 116.24 \\
degree 5 (unsatisfied) & 50 & 100 & 150 & 200 & 250 & 300 \\
\hline MTS & 0.72 & 2.81 & 6.50 & 12.45 & 19.45 & 29.58 \\
PMTS (0) & 0.83 & 4.23 & 8.51 & 14.89 & 25.33 & 37.79 \\
PMTS (1) & 1.79 & 9.25 & 29.66 & 56.53 & 69.69 & 83.31 \\
PMTS (5) & 2.89 & 7.60 & 20.14 & 42.45 & 64.86 & 89.23 \\
PMTS (20) & 1.21 & 6.87 & 20.75 & 40.51 & 47.54 & 97.75
\end{tabular}

\footnotetext{
3 Standard deviation: 40.29

4 Standard deviation: 47.56

5 Standard deviation: 197.36

6 Standard deviation: 96.79
} 


\section{Conclusion}

We have introduced an extension of modal transition systems called PMTS for parametric systems. The formalism is general enough to capture several features missing in the other extensions, while at the same time it offers an easy to understand semantics and a natural notion of modal refinement that specialises to the wellknown refinements already studied on the subclasses of PMTS. We provided a comprehensive overview of complexity of refinement checking on PMTS and its subclasses, showed a direct encoding of the problem into QBF and discussed its applicability on preliminary experiments with random graphs. The conclusion we can draw is that the refinement checking scales well with the growing number of parameters but it is more sensitive to the degree of nondeterminism. A more thorough experimental evaluation on larger case studies is left as future work.

Acknowledgements We would like to thank to Sebastian Bauer for suggesting the traffic light example and for allowing us to use his figure environments. The research leading to the results in this article has received funding from the European Union Seventh Framework Programme (FP7/2007-2013) under Grant Agreement nr. 318490 (SENSATION) and Grant Agreement nr. 601148 (CASSTING), from the Sino-Danish Basic Research Center IDEA4CPS funded by the Danish National Research Foundation and the National Science Foundation China. The research was further funded in part by the European Research Council (ERC) under the grant agreement 267989 (QUAREM), by the Austrian Science Fund (FWF) project S11402-N23 (RiSE) and by the Czech Science Foundation grant No. P202/12/G061. Nikola Beneš has been supported by the MEYS project No. CZ.1.07/2.3.00/30.0009 Employment of Newly Graduated Doctors of Science for Scientific Excellence.

\section{References}

1. Aceto, L., Fábregas, I., de Frutos-Escrig, D., Ingólfsdóttir, A., Palomino, M.: Graphical representation of covariant-contravariant modal formulae. In: EXPRESS, pp. 1-15 (2011)

2. de Alfaro, L., Henzinger, T.A.: Interface automata. In: ESEC / SIGSOFT FSE, pp. 109-120 (2001)

3. Alur, R., Henzinger, T.A., Kupferman, O., Vardi, M.Y.: Alternating refinement relations. In: CONCUR, pp. 163-178 (1998)

4. Antonik, A., Huth, M., Larsen, K.G., Nyman, U., Wasowski, A.: 20 years of modal and mixed specifications. Bulletin of the EATCS no. 95 pp. 94-129 (2008)

5. Antonik, A., Huth, M., Larsen, K.G., Nyman, U., Wasowski, A.: Complexity of decision problems for mixed and modal specifications. In: Proceedings of the 11th International Conference on Foundations of Software Science and Computation Structures (FOSSACS'08), LNCS, vol. 4962, pp. 112-126 (2008)

6. Balcazar, J.L., Gabarró, J., Santha, M.: Deciding bisimilarity is P-complete. Formal aspects of computing 4(6 A), 638-648 (1992)

7. Bauer, S.S., Fahrenberg, U., Juhl, L., Larsen, K.G., Legay, A., Thrane, C.R.: Quantitative refinement for weighted modal transition systems. In: MFCS, LNCS, vol. 6907, pp. 60-71. Springer (2011)

8. Beneš, N., Černá, I., Křetínský, J.: Disjunctive modal transition systems and generalized LTL model checking. Technical report FIMU-RS-2010-12, Faculty of Informatics, Masaryk University, Brno (2010) 
9. Beneš, N., Delahaye, B., Fahrenberg, U., Křetínský, J., Legay, A.: HennessyMilner logic with greatest fixed points as a complete behavioural specification theory. In: P.R. D'Argenio, H.C. Melgratti (eds.) CONCUR, Lecture Notes in Computer Science, vol. 8052, pp. 76-90. Springer (2013)

10. Beneš, N., Křetínský, J., Larsen, K.G., Møller, M.H., Srba, J.: Parametric modal transition systems. Technical report FIMU-RS-2011-03, Faculty of Informatics, Masaryk University, Brno (2011)

11. Beneš, N., Křetínský, J., Larsen, K.G., Srba, J.: EXPTIME-completeness of thorough refinement on modal transition systems. Inf. Comput. 218, 54-68 (2012)

12. Beneš, N., Křetínský, J.: Process algebra for modal transition systemses. In: L. Matyska, M. Kozubek, T. Vojnar, P. Zemcík, D. Antos (eds.) MEMICS, OASICS, vol. 16, pp. 9-18. Schloss Dagstuhl - Leibniz-Zentrum fuer Informatik, Germany (2010)

13. Beneš, N., Křetínský, J., Larsen, K., Srba, J.: EXPTIME-completeness of thorough refinement on modal transition systems. Information and Computation 218, 54-68 (2012)

14. Beneš, N., Křetínský, J., Larsen, K.G., Møller, M.H., Srba, J.: Parametric modal transition systems. In: ATVA, pp. 275-289 (2011)

15. Beněs, N., Křetínský, J., Larsen, K.G., Møller, M.H., Srba, J.: Dual-priced modal transition systems with time durations. In: LPAR, pp. 122-137 (2012)

16. Beneš, N., Křetínský, J., Larsen, K.G., Srba, J.: Checking thorough refinement on modal transition systems is EXPTIME-complete. In: Theoretical Aspects of Computing - ICTAC 2009, 6th International Colloquium. Proceedings, LNCS, vol. 5684. Springer (2009)

17. Beneš, N., Křetínský, J., Larsen, K.G., Srba, J.: On determinism in modal transition systems. Theoretical Computer Science 410(41), 4026-4043 (2009)

18. Beneš, N., Křetínský, J., Larsen, K.G., Srba, J.: On determinism in modal transition systems. Theor. Comput. Sci. 410(41), 4026-4043 (2009)

19. Beneš, N., Cerná, I., Křetínský, J.: Modal transition systems: Composition and LTL model checking. In: ATVA, pp. 228-242 (2011)

20. Bertrand, N., Legay, A., Pinchinat, S., Raclet, J.B.: Modal event-clock specifications for timed component-based design. Sci. Comput. Program. 77(12), 1212-1234 (2012). DOI 10.1016/j.scico.2011.01.007

21. Boudol, G., Larsen, K.G.: Graphical versus logical specifications. In: CAAP, pp. 57-71 (1990)

22. Boudol, G., Larsen, K.G.: Graphical versus logical specifications. Theor. Comput. Sci. 106(1), 3-20 (1992)

23. Caillaud, B., Delahaye, B., Larsen, K.G., Legay, A., Pedersen, M.L., Wasowski, A.: Compositional design methodology with constraint markov chains. In: QEST, pp. 123-132 (2010)

24. Campetelli, A., Gruler, A., Leucker, M., Thoma, D.: Don't Know for multivalued systems. In: ATVA, pp. 289-305 (2009)

25. Čerāns, K., Godskesen, J.C., Larsen, K.G.: Timed modal specification - theory and tools. In: CAV, pp. 253-267 (1993)

26. Dams, D., Gerth, R., Grumberg, O.: Abstract interpretation of reactive systems. ACM Trans. Program. Lang. Syst. 19(2), 253-291 (1997)

27. Dams, D., Namjoshi, K.S.: The existence of finite abstractions for branching time model checking. In: LICS, pp. 335-344 (2004) 
28. David, A., Larsen, K.G., Legay, A., Nyman, U., Wasowski, A.: ECDAR: An environment for compositional design and analysis of real time systems. In: ATVA, pp. 365-370 (2010)

29. Fecher, H., Schmidt, H.: Comparing disjunctive modal transition systems with an one-selecting variant. J. of Logic and Alg. Program. 77(1-2), 20-39 (2008)

30. Fecher, H., Steffen, M.: Characteristic mu-calculus formulas for underspecified transition systems. ENTCS 128(2), 103-116 (2005)

31. Godefroid, P., Huth, M., Jagadeesan, R.: Abstraction-based model checking using modal transition systems. In: Proc. CONCUR'01, LNCS, vol. 2154, pp. 426-440. Springer (2001)

32. Godefroid, P., Nori, A.V., Rajamani, S.K., Tetali, S.: Compositional may-must program analysis: unleashing the power of alternation. In: POPL, pp. $43-56$ (2010)

33. Gruler, A., Leucker, M., Scheidemann, K.D.: Modeling and model checking software product lines. In: G. Barthe, F.S. de Boer (eds.) FMOODS, Lecture Notes in Computer Science, vol. 5051, pp. 113-131. Springer (2008)

34. Huth, M., Jagadeesan, R., Schmidt, D.A.: Modal transition systems: A foundation for three-valued program analysis. In: Proc. of ESOP'01, LNCS, vol. 2028, pp. 155-169. Springer (2001)

35. Jacobs, B., Poll, E.: A logic for the java modeling language JML. In: FASE, pp. 284-299 (2001)

36. Juhl, L., Larsen, K.G., Srba, J.: Modal transition systems with weight intervals. J. Log. Algebr. Program. 81(4), 408-421 (2012)

37. Křetínský, J., Sickert, S.: MoTraS: A tool for modal transition systems and their extensions. In: D.V. Hung, M. Ogawa (eds.) ATVA, Lecture Notes in Computer Science, vol. 8172, pp. 487-491. Springer (2013). Tool accessible at https://www7.in.tum.de/ kretinsk/motras.html

38. Křetínský, J., Sickert, S.: On refinements of Boolean and parametric modal transition systems. In: Z. Liu, J. Woodcock, H. Zhu (eds.) ICTAC, Lecture Notes in Computer Science, vol. 8049, pp. 213-230. Springer (2013)

39. Křetínský, J., Sickert, S.: On refinements of Boolean and parametric modal transition systems. CoRR abs/1304.5278 (2013)

40. Larsen, K.G., Nyman, U., Wasowski, A.: Modal I/O automata for interface and product line theories. In: ESOP, pp. 64-79 (2007)

41. Larsen, K.G., Nyman, U., Wasowski, A.: On modal refinement and consistency. In: Proc. of CONCUR'07, LNCS, vol. 4703, pp. 105-119. Springer (2007)

42. Larsen, K.G., Thomsen, B.: A modal process logic. In: LICS, pp. 203-210. IEEE Computer Society (1988)

43. Larsen, K.G., Xinxin, L.: Equation solving using modal transition systems. In: LICS, pp. 108-117. IEEE Computer Society (1990)

44. Liskov, B., Wing, J.M.: A behavioral notion of subtyping. ACM Trans. Program. Lang. Syst. 16(6), 1811-1841 (1994)

45. Lüttgen, G., Vogler, W.: Modal interface automata. Logical Methods in Computer Science 9(3) (2013). DOI 10.2168/LMCS-9(3:4)2013. URL http: //dx.doi.org/10.2168/LMCS-9(3:4) 2013

46. Lynch, N.: I/O automata: A model for discrete event systems. In: 22nd Annual Conference on Information Sciences and Systems, pp. 29-38. Princeton University (1988) 
47. Namjoshi, K.S.: Abstraction for branching time properties. In: CAV, pp. 288-300 (2003)

48. Nanz, S., Nielson, F., Nielson, H.R.: Modal abstractions of concurrent behaviour. In: Proc. of SAS'08, LNCS, vol. 5079, pp. 159-173. Springer (2008)

49. Papadimitriou, C.H.: Computational complexity. Addison-Wesley Publishing Co., Inc., Reading, MA, USA (1994)

50. Raclet, J.B.: Quotient de spécifications pour la réutilisation de composants. Ph.D. thesis, Université de Rennes I (2007). (In French)

51. Raclet, J.B., Badouel, E., Benveniste, A., B.Caillaud, Legay, A., Passerone, R.: A modal interface theory for component-based design. Fundamenta Informaticae 108(1-2), 119-149 (2011)

52. Raclet, J.B., Badouel, E., Benveniste, A., Caillaud, B., Passerone, R.: Why are modalities good for interface theories? In: ACSD, pp. 119-127. IEEE (2009)

53. Sawa, Z., Jančar, P.: Behavioural equivalences on finite-state systems are PTIME-hard. Computing and informatics 24(5), 513-528 (2005)

54. Uchitel, S., Chechik, M.: Merging partial behavioural models. In: Proc. of FSE'04, pp. 43-52. ACM (2004) 